\title{
The CDK4/6-EZH2 pathway is a potential therapeutic target for psoriasis
}

\author{
Anne Müller, ${ }^{1}$ Antje Dickmanns, ${ }^{2}$ Claudia Resch, ${ }^{1}$ Knut Schäkel, ${ }^{3}$ Stephan Hailfinger, ${ }^{1,4}$ Matthias Dobbelstein, ${ }^{2}$ \\ Klaus Schulze-Osthoff, ${ }^{1,5}$ and Daniela Kramer ${ }^{1}$
}

IInterfaculty Institute for Biochemistry, University of Tübingen, Tübingen, Germany. ²Institute of Molecular Oncology, Göttingen Center of Molecular Biosciences (CZMB), University of Cöttingen, Cöttingen, Cermany. ${ }^{3}$ Department of Dermatology, Heidelberg University Hospital, Heidelberg, Germany. ${ }^{4}$ Cluster of Excellence iFIT (EXC 2180), Image-Guided and Functionally Instructed Tumor Therapies, University of Tübingen, Tübingen, Germany. ${ }^{5}$ German Cancer Consortium (DKTK) and German Cancer Research Center (DKFZ), Heidelberg, Germany.

\begin{abstract}
Psoriasis is a frequent, inflammatory skin disease characterized by keratinocyte hyperproliferation and a disease-related infiltration of immune cells. Here, we identified a novel proinflammatory signaling pathway driven by cyclin-dependent kinase 4 (CDK4) and CDK6 and the methyltransferase EZH2 as a valid target for psoriasis therapy. Delineation of the pathway revealed that CDK4/6 phosphorylated EZH2 in keratinocytes, thereby triggering a methylation-induced activation of STAT3. Subsequently, active STAT3 resulted in the induction of $І \kappa \mathrm{B} \zeta$, which is a key proinflammatory transcription factor required for cytokine synthesis in psoriasis. Pharmacological or genetic inhibition of CDK4/6 or EZH2 abrogated psoriasis-related proinflammatory gene expression by suppressing ІкB $\zeta$ induction in keratinocytes. Importantly, topical application of CDK4/6 or EZH2 inhibitors on the skin was sufficient to fully prevent the development of psoriasis in various mouse models by suppressing STAT3-mediated IKB $\zeta$ expression. Moreover, we found a hyperactivation of the CDK4/6-EZH2 pathway in human and mouse psoriatic skin lesions. Thus, this study not only identifies a novel psoriasis-relevant proinflammatory pathway, but also proposes the repurposing of CDK4/6 or EZH2 inhibitors as a new therapeutic option for patients with psoriasis.
\end{abstract}

\section{Introduction}

Psoriasis is a mixed autoimmune and autoinflammatory skin disease, affecting $2 \%$ to $3 \%$ of the population worldwide. Psoriatic skin lesions are characterized by keratinocyte hyperproliferation and a massive infiltration of immune cells, such as neutrophils, macrophages, and Th17 cells (1). The cytokine families IL-17 and IL-36 have been identified as key factors driving the establishment of psoriatic plaques (2). Therefore, state-of-the-art therapies comprise neutralizing antibodies against IL-17 $(3,4)$, while IL-36 antagonists are currently tested in clinical trials (5). Although psoriasis therapy with neutralizing antibodies is very effective, disadvantages comprise high costs, difficult application routes, systemic side effects such as upper respiratory tract infections, and long-term therapy resistance due to the development of antidrug antibodies $(6,7)$. Therefore, effective new therapy approaches against psoriasis are needed.

Previously, IкB $\zeta$, encoded by the gene $N F K B I Z$, has been identified as a key regulator of transcription in psoriasis $(8,9)$. $\mathrm{I} \kappa \mathrm{B} \zeta$ represents an atypical member of the I $\mathrm{B}$ family that is inducibly expressed and then accumulates in the nucleus, leading to the activation or repression of a selective subset of NF- $\kappa \mathrm{B}$ target genes (10). Especially in keratinocytes, IL-17A, alone or even

Conflict of interest: DK, AM, and KSO filed patent applications for the treatment of psoriasis using CDK4/6 and EZH2 inhibitors at the European Patent Office (19200621.1 and 19200622.9, "Agent for the treatment of psoriasis"). Copyright: (c) 2020, American Society for Clinical Investigation.

Submitted: October 11, 2019; Accepted: July 14, 2020; Published: September 28, 2020. Reference information: / Clin Invest. 2020;130(11):5765-5781.

https://doi.org/10.1172/JCl134217. more potently in combination with TNF- $\alpha$ as well as IL-36 cytokines, triggers a NF- $\mathrm{KB}$ - and STAT3-dependent transcriptional upregulation of $\mathrm{I} \kappa \mathrm{B} \zeta$ expression (9). Subsequently, I $\mathrm{I} B \zeta$ induces a subset of IL-36- and IL-17-responsive target genes in keratinocytes, including CXCL2, CXCL5, CXCL8, LCN2, DEFB4, or IL1B, which all have already been implicated in the pathogenesis of psoriasis (2). How $\mathrm{I} \kappa \mathrm{B} \zeta$ regulates these downstream target genes remains elusive. It is assumed that $\mathrm{I} \kappa \mathrm{B} \zeta$ recruits epigenetic modifiers, such as TET2 or the SWI/SNF complex, to the promoter sites of its target genes, leading to a change in DNA methylation or nucleosome remodeling $(11,12)$.

In agreement with its role as a key regulator of psoriasis-related gene expression, $\mathrm{I} \kappa \mathrm{B} \zeta$-deficient mice are completely protected against imiquimod-mediated (IMQ-mediated) or IL-36-mediated psoriasis-like skin inflammation $(8,9)$. Moreover, human psoriatic skin lesions are characterized by an upregulated expression of $\operatorname{I} \kappa \zeta \zeta(8,9)$. Altogether, these findings validate $\mathrm{I} \kappa \mathrm{B} \zeta$ as an attractive new therapeutic target in psoriasis. $A s \mathrm{I} \kappa \mathrm{B} \zeta$ lacks any enzymatic activity, it is difficult to develop direct $\mathrm{I} \kappa \mathrm{B} \zeta$ inhibitors (13). Therefore, small molecule inhibitors blocking the induction or downstream function of $\mathrm{I} \kappa \mathrm{B} \zeta$ could represent an alternative strategy for targeting $\mathrm{I} \kappa \mathrm{B} \zeta$ in psoriasis.

CDK4 and CDK6, in complex with cyclin D1, cyclin D2, or cyclin D3, represent well known cell-cycle regulating kinases that can phosphorylate $\mathrm{RB}$, leading to the release of $\mathrm{E} 2 \mathrm{~F}$ transcription factors and G1-S cell cycle transition (14). Consistently, amplification of CDK4 and CDK6 as well as overexpression of cyclin D proteins are frequently observed events in cancer, leading to the excessive proliferation of tumor cells $(15,16)$. ATP-competitive CDK4/6 inhibitors, such 

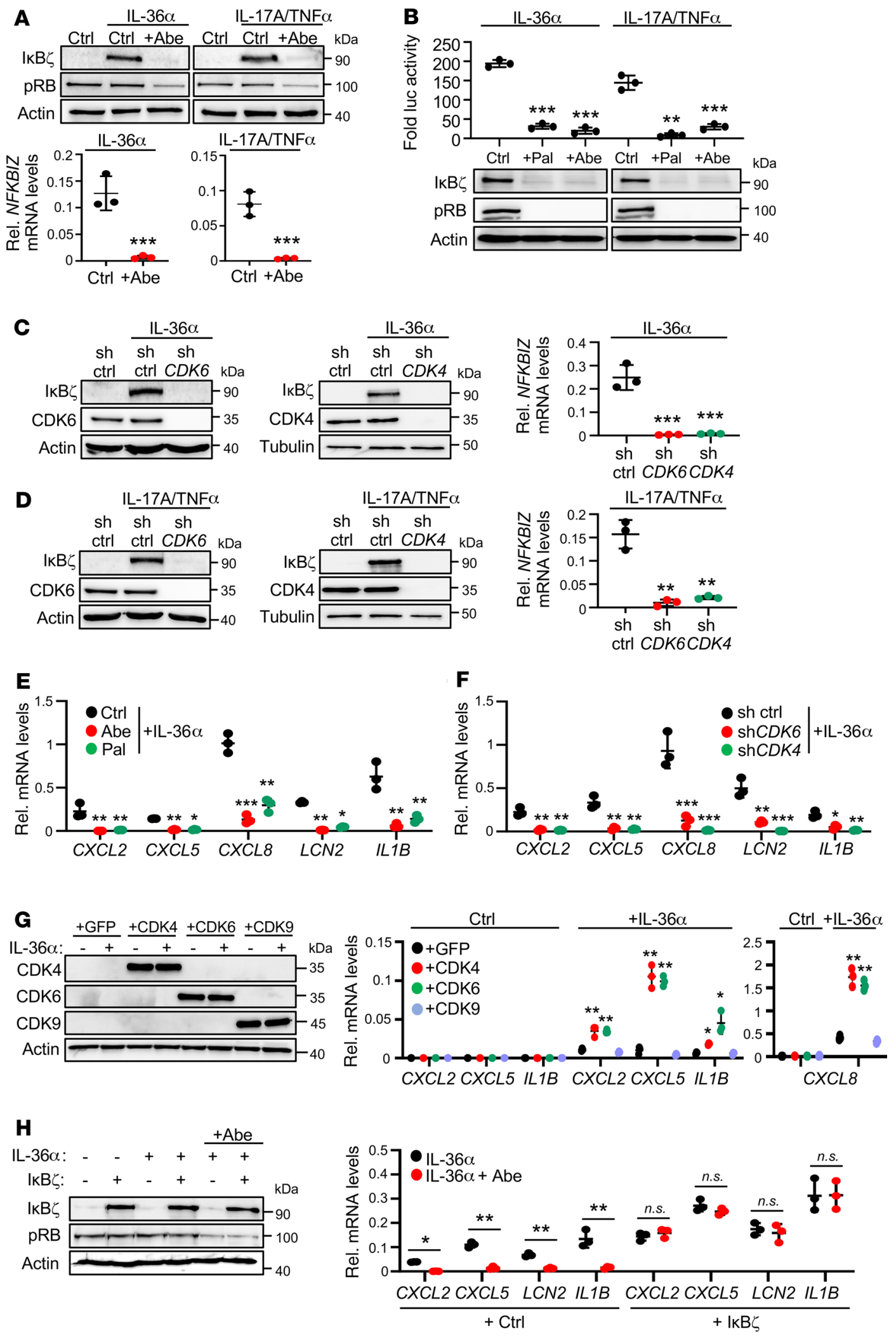
Figure 1. CDK4 and CDK6 regulate the expression of IKB $\zeta$ and its proinflammatory target genes in IL-36 $\alpha$ - and IL-17A/TNF- $\alpha$-stimulated keratinocytes. (A) Human primary keratinocytes were treated for 1 hour with $100 \mathrm{ng} / \mathrm{mL}$ IL-36 $\alpha$ or $200 \mathrm{ng} / \mathrm{mL}$ IL-17A and $10 \mathrm{ng} / \mathrm{mL}$ TNF- $\alpha$. The CDK4/6 inhibitor abemaciclib (Abe) or an ethanol vehicle control (Ctrl) were added in parallel. Phosphorylation of RB (pRB) served as a control for CDK4/6 inhibition, and actin as a loading control. Relative mRNA levels of

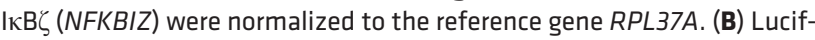
erase assay of I $\kappa \zeta \zeta$ (NFKBIZ) promoter activity in $\mathrm{HaCaT}$ cells that were cytokine-stimulated for 24 hours in the presence or absence of the CDK4/6 inhibitors abemaciclib or palbociclib (Pal). Relative luciferase (luc) activity was normalized to an internal Renilla luciferase control that was transfected in parallel. Endogenous protein levels were analyzed as input controls by immunoblotting (bottom). (C and D) CDK4 and CDK6 were depleted in primary human keratinocytes by lentiviral transduction of shRNA. Ctrl shRNA- or CDK4/6 shRNA-depleted cells were treated with (C) IL-36 $\alpha$ or (D) IL-17A/TNF- $\alpha$, similar as in A. (E and F) Human primary keratinocytes were stimulated with IL-36 $\alpha$ as in A. (E) Cytokine gene expression in CDK4/6 inhibitor-treated cells. (F) Relative gene expression levels in IL-36 $\alpha$-treated control or CDK4/6-depleted cells. (G) Transient overexpression of CDK4, CDK6, or CDK9 in HaCaT cells, treated for 1 hour with $100 \mathrm{ng} /$ $\mathrm{mL}$ IL-36 $\alpha$. (H) Cytokine gene expression in IL-36 $\alpha$-treated primary keratinocytes overexpressing $I \kappa B \zeta$ in the presence or absence of abemaciclib. For all analyses, $n=3 \pm S D$. Significance was calculated using a 1-way ANOVA for multiple groups and a 2-tailed Student's $t$ test for comparing 2 groups: ${ }^{*} P<0.05 ;{ }^{* *} P<0.01$; ${ }^{* *} P<0.001$.

as palbociclib and abemaciclib, have been developed for anticancer therapy and were recently approved for treatment of breast cancer patients (17). Interestingly, common side effects of a CDK4/6 inhibitor therapy constitute neutropenia and leukopenia $(17,18)$. Moreover, it was found that CDK4/6 inhibition modulates immune cell functions in kinase-dependent or -independent manners (19-22). Mechanistically, it is assumed that these atypical functions of CDK4 and CDK6 derive from their recently discovered role as cofactors for immune regulatory transcription factors (23-25). CDK6, especially, can colocalize at promoter regions of a subset of NF- $\kappa \mathrm{B}$, STAT3, or AP1 target genes, thereby changing the DNA-binding properties or activity of these transcription factors.

We screened for small-molecule inhibitors of $\mathrm{I} \kappa \mathrm{B} \zeta$ action in keratinocytes and identified CDK4/6 inhibitors as potent suppressors of IL-36- and IL-17A/TNF- $\alpha$-mediated I $\kappa$ B $\zeta$ expression. Mechanistically, CDK4/6 inhibitors suppressed the activity of STAT3, which was identified as a major transcriptional regulator

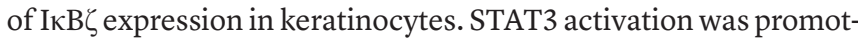
ed by CDK4/6-mediated phosphorylation of the methyltransferase EZH2, triggering the subsequent methylation of STAT3 and induction of $\mathrm{I} \kappa \mathrm{B} \zeta$ expression. Importantly, topical administration of CDK4/6 or EZH2 inhibitors on the skin completely prevented experimental psoriasis by suppressing STAT3 activation and consequently, I $\mathrm{K} \mathrm{B} \zeta$ expression in keratinocytes. Moreover, as cyclin D2, cyclin D3, and EZH2 were found to be overexpressed in human psoriatic skin lesions, we propose repurposing CDK4/6 and EZH2 inhibitors for topical skin treatment of patients with psoriasis.

\section{Results}

$C D K 4 / 6$ inhibitors suppress the expression of $I \kappa B \zeta$ and $I \kappa B \zeta$-dependent, proinflammatory genes in IL-36 $\alpha$ - and IL-17A/TNF- $\alpha$-stimulated keratinocytes. $\mathrm{I} \kappa \mathrm{B} \zeta$ represents an attractive therapeutic target for psoriasis. However, due to a lack of enzyme activity, direct inhibition of $\mathrm{I} \kappa \mathrm{B} \zeta$ is not feasible. Key regulators in psoriasis constitute IL-17 and IL-36 family members $(26,27)$, which predominantly trigger a proinflammatory response in keratinocytes that is dependent on $\mathrm{I} \kappa \mathrm{B} \zeta(8,9)$. Thus, we screened for small-molecule inhibitors that are able to block induction of $\mathrm{I} \mathrm{B} \zeta$ expression in response to either IL-36 $\alpha$ or IL-17A. Previously, it was shown that IL-17induced $\mathrm{I} \kappa \mathrm{B} \zeta$ expression is strongly increased in combination with TNF- $\alpha(8,9)$. Intriguingly, we found that 2 CDK $4 / 6$ inhibitors, abemaciclib (Figure 1A and Supplemental Figure 1A; supplemental material available online with this article; https://doi.org/10.1172/ JCI134217DS1) and palbociclib (Supplemental Figure 1B), completely blocked IL-36 $\alpha$ - or IL-17A/TNF- $\alpha$-mediated induction of $\mathrm{I} \kappa \mathrm{B} \zeta$ expression in primary human keratinocytes. Moreover, we observed similar effects in response to IL-36 $\gamma$, IL-1 $\beta$, or the TLR ligands flagellin and poly(I:C) (Supplemental Figure 1, C and D), thereby revealing a conservation of this pathway in keratinocytes.

To explore whether these effects were due to a CDK4/6 inhibitor-mediated G1 cell cycle arrest, we repeated the experiments in synchronized and single cell cycle phase-arrested keratinocytes. IL-36 $\alpha$ treatment triggered I $\mathrm{B}$ B $\zeta$ induction in all phases of the cell cycle; induction was completely suppressed by abemaciclib (Supplemental Figure 1E). Moreover, depletion of $R B$ by RNA interference did not influence IL-36-mediated induction or abe-

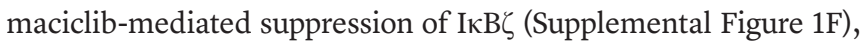
thereby clearly indicating that the effect of CDK4/6 inhibitors on $\mathrm{I} \kappa \mathrm{B} \zeta$ expression was independent of their ability to trigger cell cycle arrest. Instead, we revealed that CDK4/6-dependent induction of $\mathrm{I} \kappa \mathrm{B} \zeta$ was mediated at the transcriptional level, as palbociclib and abemaciclib treatment abrogated the expression of a luciferase

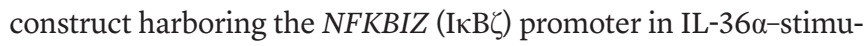
lated $\mathrm{HaCaT}$ cells (Figure 1B). Interestingly, also shRNA-mediated depletion of CDK4 or CDK6 was sufficient to suppress IL-36 $\alpha^{-}$or IL-17A/TNF- $\alpha$-dependent expression of $\mathrm{I} \kappa \mathrm{B} \zeta$ in human primary keratinocytes, thereby excluding off-target effects of the applied inhibitors (Figure 1, C and D). Accordingly, IкB $\zeta$-dependent target genes, such as CXCL2, CXCL5, or CXCL8, were strongly downregulated in IL-36 $\alpha$ - and CDK4/6 inhibitor-treated keratinocytes (Figure 1E), as well as in CDK4- or CDK6-deficient cells (Figure $1 \mathrm{~F})$, whereas other $\mathrm{NF}-\kappa \mathrm{B}$-dependent but $\mathrm{I} \kappa \mathrm{B} \zeta$-independent genes, such as NFKBIA or TNF, remained unaffected (Supplemental Figure $1 \mathrm{G})$. Similar effects of pharmacological or shRNA-mediated inhibition of CDK4/ 6 were obtained in IL-17A and TNF- $\alpha$-stimulated cells (Supplemental Figure 1, H and I). CDK4/6 inhibitors have the potential to inhibit CDK9 kinase activity, although much higher concentrations are needed (17). To rule out effects deriving from the suppression of CDK9 activity, we transiently overexpressed CDK4, CDK6, or CDK9 in HaCaT cells and analyzed IL-36 $\alpha$-mediated gene expression. Overexpression of CDK4 and CDK6, but not CDK9, could increase IL-36 $\alpha$-mediated, IкB $\zeta$ dependent target gene expression in keratinocytes, thereby further confirming the specificity of CDK4 and CDK6 in regulating proinflammatory target gene expression in keratinocytes (Figure $1 G$ ).

We hypothesized that CDK4 and CDK6 are not involved in the direct regulation of $\mathrm{I} \kappa \mathrm{B} \zeta$ target gene expression but rather trigger the expression of $\mathrm{I} \kappa \mathrm{B} \zeta$, which in turn induces a secondary, I $\mathrm{K} \mathrm{B} \zeta$-dependent gene expression in stimulated keratinocytes. 


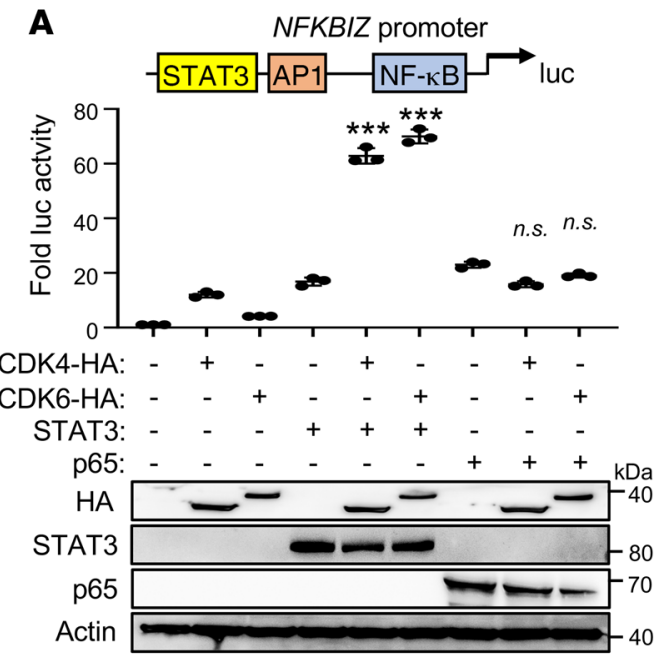

B

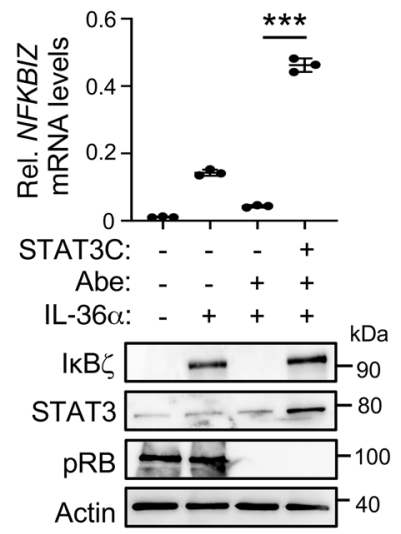

C

D

\section{NFKBIZ promoter}
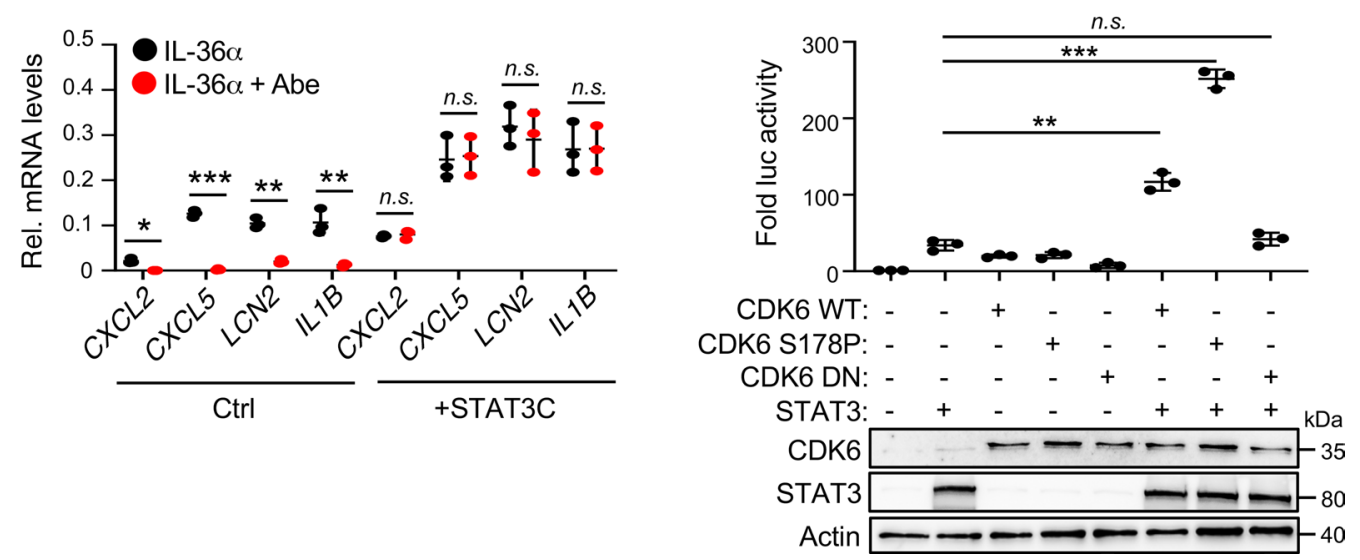

Figure 2. STAT3 mediates CDK4/6-dependent IKB $\zeta$ induction in keratinocytes. (A) Luciferase assay of the NFKBIZ promoter in HEK293T cells after transient expression of CDK4, CDK6, STAT3, or p65, alone or in combination. The plasmid amounts for STAT3 (200 ng) and p65 (70 ng) were adjusted to achieve similar luciferase activity in the absence of CDK4/6 expression. Overexpression of the HA-tagged CDK4 and CDK6 proteins was detected using a HA-antibody. (B) Primary human keratinocytes with a transient overexpression of hyperactive STAT3 (STAT3C) were treated for 1 hour with $100 \mathrm{ng} / \mathrm{mL}$ IL-36 $\alpha$ and abemaciclib (Abe). NFKBIZ mRNA levels normalized to RPL37A. Immunoblot analysis of STAT3C overexpression and CDK4/6 inhibition. (C) IKB $\zeta$ target gene expression in STAT3C-overexpressing primary keratinocytes. Treatment as in B. (D) Luciferase activity assay of the NFKBIZ promoter in HEK293T cells overexpressing STAT3 alone or in combination with WT CDK6 (wt), hyperactive CDK6 (S178P), or a kinase-dead CDK6 mutant (CDK6 DN). For all analyses, $n=3 \pm$ SD. Significance was calculated using a 1-way ANOVA for multiple groups and a 2-tailed Student's $t$ test for comparing 2 groups: ${ }^{*} P<0.05 ;{ }^{* *} P<0.01 ;{ }^{* * *} P<0.001$.

To test this hypothesis, we transiently overexpressed ІкB $\zeta$ in IL-36 $\alpha$ - or IL17A/TNF- $\alpha$-stimulated primary human keratinocytes in the presence or absence of abemaciclib (Figure $1 \mathrm{H}$ and Supplemental Figure 1J). In this setup, exogenous overexpression of IкB $\zeta$

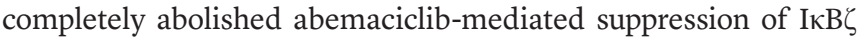
target gene expression, thereby validating CDK4/6-mediated transcriptional upregulation of $\mathrm{I} \kappa \mathrm{B} \zeta$ as a prerequisite for $\mathrm{CDK} 4 / 6$ dependent, proinflammatory gene expression in keratinocytes.

CDK4/6-dependent induction of I $\kappa \zeta \zeta$ expression is mediated by STAT3 in a cyclin-dependent manner. Beside their known involvement in cell cycle regulation, CDK4 and CDK6 have been described to function as transcriptional cofactors for STAT3, NF- $\mathrm{B}$, or AP-1 (23-25). As we revealed a CDK4/6-dependent induction of $\mathrm{I} \kappa \mathrm{B} \zeta$ on the transcriptional level, we next explored the responsible transcription factor. Of note, binding sites for all 3 transcription factors were previously identified at the NFKBIZ promoter region (9). Interestingly, expression of both CDK4 and
CDK6 increased the STAT3-mediated induction of NFKBIZ promoter activity, whereas no synergistic effects could be observed when CDK4 and CDK6 were co-overexpressed with NF- $\kappa \mathrm{B}$ p65 or cJun (Figure 2A and Supplemental Figure 2A). In agreement, deletion of the STAT3-binding site abrogated the expression of the NFKBIZ luciferase reporter in IL-36 $\alpha$-stimulated, CDK4/6-overexpressing $\mathrm{HaCaT}$ cells, whereas deletion of the NF- $\mathrm{\kappa B}$ or AP1 motif had only a minor or almost no effect (Supplemental Figure 2B). Finally, transient overexpression of a constitutively active STAT3 mutant (STAT3C) abrogated the effects of CDK4/6 inhibition on the induction of $\mathrm{I} \kappa \mathrm{B} \zeta$ (Figure $2 \mathrm{~B}$ ) and $\mathrm{I} \kappa \mathrm{B} \zeta$-dependent target gene expression in IL-36 $\alpha$-stimulated primary keratinocytes (Figure 2C), thereby validating STAT3 as the responsible transcription factor for CDK4/6-mediated effects in keratinocytes.

Previous publications reported that CDK6 acts as a cofactor for STAT3, independently of its kinase function (23). Therefore, we tested if a kinase-dead mutant of CDK6 (CDK6 DN) could 
still synergize with STAT3 in driving the expression of the NFK$B I Z$ luciferase reporter construct. Surprisingly, the kinase-dead mutant was not able to cooperate with STAT3 anymore, whereas a hyperactive version of CDK6 (CDK6 S178P) further increased the activity of the NFKBIZ promoter in a STAT3-dependent manner (Figure 2D). Accordingly, cyclin D2 and cyclin D3, which associate with CDK4/6 to activate their kinase function (14), synergized with CDK4/6 and STAT3 in activating the NFKBIZ luciferase promoter, whereas cyclin D1 failed to do so (Supplemental Figure 2, C and D). Moreover, cyclin D2 and cyclin D3 overexpression significantly elevated the expression of NFKBIZ and its target genes in IL-36 $\alpha-$ stimulated keratinocytes (Supplemental Figure 2, E and F).

Cyclin D2 and cyclin D3 levels are transcriptionally regulated by NF-кB $(28,29)$. Therefore, we hypothesized that IL-36 $\alpha$ or IL-17A/TNF- $\alpha$ stimulation results in a transient NF- $\mathrm{B}$-dependent upregulation of cyclin D2/D3, thereby explaining the cooperation of CDK4/6 and STAT3 in triggering IкB detected a rapid binding of NF- $\kappa \mathrm{B}$ p65 to the promoter regions of CCND2 and CCND3 upon stimulation of primary keratinocytes with IL-36 $\alpha$ (Supplemental Figure 2G). Consequently, IL-36 $\alpha$ stimulation led to increased expression of CCND2 and CCND3 in a p65-dependent manner (Supplemental Figure $2 \mathrm{H}$ ), thus validat-

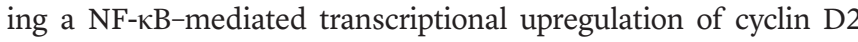
and cyclin D3 in stimulated keratinocytes. Although p65 failed to cooperate with CDK4/6 in the induction of the NFKBIZ promoter directly (Figure $2 \mathrm{~A}$ ), we hypothesized that NF- $\kappa \mathrm{B}$ participates in the induction of $\mathrm{I} \kappa \mathrm{B} \zeta$ in keratinocytes by transcriptionally upregulating cyclin D2/D3 levels, leading to activation of CDK4/6. In agreement, whereas knockdown of p65/RELA abrogated I $\mathrm{KB} \zeta$ expression in IL-36 $\alpha$-stimulated keratinocytes, exogenous overexpression cyclin D2 could fully restore the expression of NFKBIZ and its target genes in IL-36 $\alpha$-stimulated primary keratinocytes (Supplemental Figure 2I). Thus, our data imply that IL-36 $\alpha$ and IL-17A/TNF- $\alpha$ stimulation of keratinocytes first activates NF- $\mathrm{BB}$, leading to an upregulation of cyclin D2 and D3 levels. Subsequently, CDK4 and CDK6 become activated, leading to a STAT3-mediated induction of IкB $\zeta$.

CDK4 and CDK6 phosphorylate EZH2 to induce STAT3-mediated $I \kappa B \zeta$ expression. Next, we explored the mechanism of how

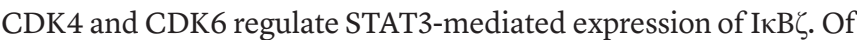
note, in chromatin immunoprecipitation (ChIP) analyses, CDK4 and CDK6 were found to localize to the NFKBIZ promoter region, which depended on the presence of STAT3 (Supplemental Figure $3 A)$. Vice versa, knockdown of CDK6 abrogated the binding of STAT3 at the NFKBIZ promoter (Supplemental Figure 3B). We reasoned that this interdependency was due to a CDK4/6-dependent regulation of STAT3 activity in keratinocytes. Accordingly, whereas the putative CDK-dependent phosphorylation site of STAT3 at threonine 727 (T727) remained unaffected (30), phosphorylation of STAT3 at tyrosine 705 (Y705), a prerequisite for STAT3 activation, was completely absent in abemaciclib-treated or CDK4/6deficient cells after stimulation with IL-36 (Figure 3, A and B). As CDK4 and CDK6 are not able to directly trigger Y705 STAT3 phosphorylation, we assumed that CDK4/6-mediated activation of STAT3 might be exerted through an altered availability or activity of a cofactor needed for STAT3 activation in keratinocytes.

Previously, EZH2, a methyltransferase that directs H3K27me3 in conjunction with the PRC2 complex, was found to be important in the differentiation and function of keratinocytes (31-33). Moreover, it was revealed that EZH2 can methylate STAT3 at lysine 49, 140 , or 180 , thereby modulating STAT3 activity by affecting the subcellular localization or phosphorylation status of STAT3 at tyrosine 705 (34-36). We hypothesized that CDK4/6 might phosphorylate EZH2 in keratinocytes, thus enabling EZH2-mediated methylation and activation of STAT3. Pull-down assays in HEK293T cells validated an interaction of CDK4 and CDK6 with EZH2 (Supplemental Figure 3C). In agreement, EZH2 inhibition by EPZ6438 or shRNA-mediated depletion of EZH2 inhibited STAT3 activation and induction of I $\kappa$ B $\zeta$ in IL-36 $\alpha$ - or IL-17A/TNF- $\alpha$-stimulated keratinocytes (Figure 3C and Supplemental Figure 3D). Furthermore, pharmacological inhibition or depletion of EZH2 effectively prevented IкB $\zeta$-dependent target gene expression in IL-36 $\alpha$-treated keratinocytes (Supplemental Figure 3E). Thus, we hypothesized that CDK4/6 phosphorylates EZH2 in keratinocytes, thereby regulating EZH2-dependent activation of STAT3.

In primary human keratinocytes, expression of EZH2 itself was induced by IL-36 $\alpha$ (Figure 3D), in line with its previous identification as an NF-אB-regulated target gene (37). Of note, EZH2 harbors 2 potential CDK phosphorylation sites at threonine 345 and 487 (Supplemental Figure 3F), which were previously shown to be phosphorylatable by CDK1/2, thereby modifying EZH2 function (38-40). Indeed, phosphorylation of EZH2 at threonine 345 (T345), but not at threonine 487 (T487) was induced in IL-36 $\alpha$ - or IL-17A/TNF- $\alpha$-treated keratinocytes, whereas abemaciclib treatment or CDK4/6 depletion completely abrogated this inducible EZH2 phosphorylation (Figure 3D and Supplemental Figure 3G). Moreover, phosphorylated EZH2 (T345) preferentially interacted with STAT3 in HaCaT cells, whereas CDK4/6 inhibition did not only abrogate the phosphorylation of EZH2 but also its interaction with STAT3 (Figure 3E).

These data suggest that CDK4/6-mediated phosphorylation of EZH2 at threonine 345 represents a regulatory switch, leading to the interaction of EZH2 with STAT3 and subsequent STAT3 activation. Accordingly, whereas WT EZH2 synergistically induced the expression of the NFKBIZ luciferase promoter in cooperation with CDK4/6 and STAT3, an EZH2 mutant lacking the CDK4/6-directed phosphorylation site (EZH2 T345A) abrogated CDK4/6- and STAT3-mediated NFKBIZ promoter-driven luciferase expression (Figure 3F). Furthermore, transient expression of a phospho-mimicking EZH2 (T345D) version could override abemaciclib-mediated suppression of $\mathrm{I} \kappa \mathrm{B} \zeta$ induction and I $\mathrm{\kappa B} \zeta$ target gene expression in IL-36 $\alpha$-stimulated primary keratinocytes (Figure $3 G$ ), whereas transient overexpression of I $\mathrm{B} \zeta$ abolished the effects of the pharmacological EZH2 inhibitor (Figure 3H). Finally, also STAT3C overexpression could override target gene expression defects in IL-36 $\alpha$-stimulated, EZH2-depleted keratinocytes (Supplemental Figure 3H), thereby validating STAT3 as the main target for suppression of gene expression in EZH2 inhibitor-treated keratinocytes. Therefore, we conclude that IL-36 $\alpha-$ and

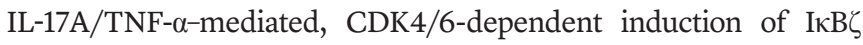
expression is mediated by phosphorylation of EZH2 at T345, thereby triggering an EZH2-dependent activation of STAT3 in keratinocytes.

CDK4/6-phosphorylated EZH2 mediates STAT3 methylation at $\mathrm{K} 180$, leading to $\mathrm{I} \kappa \mathrm{B} \zeta$ expression in keratinocytes. As reported before, EZH2 can methylate STAT3 at lysine 49, 140, or 180, thereby changing its transcription factor function or subcellular 

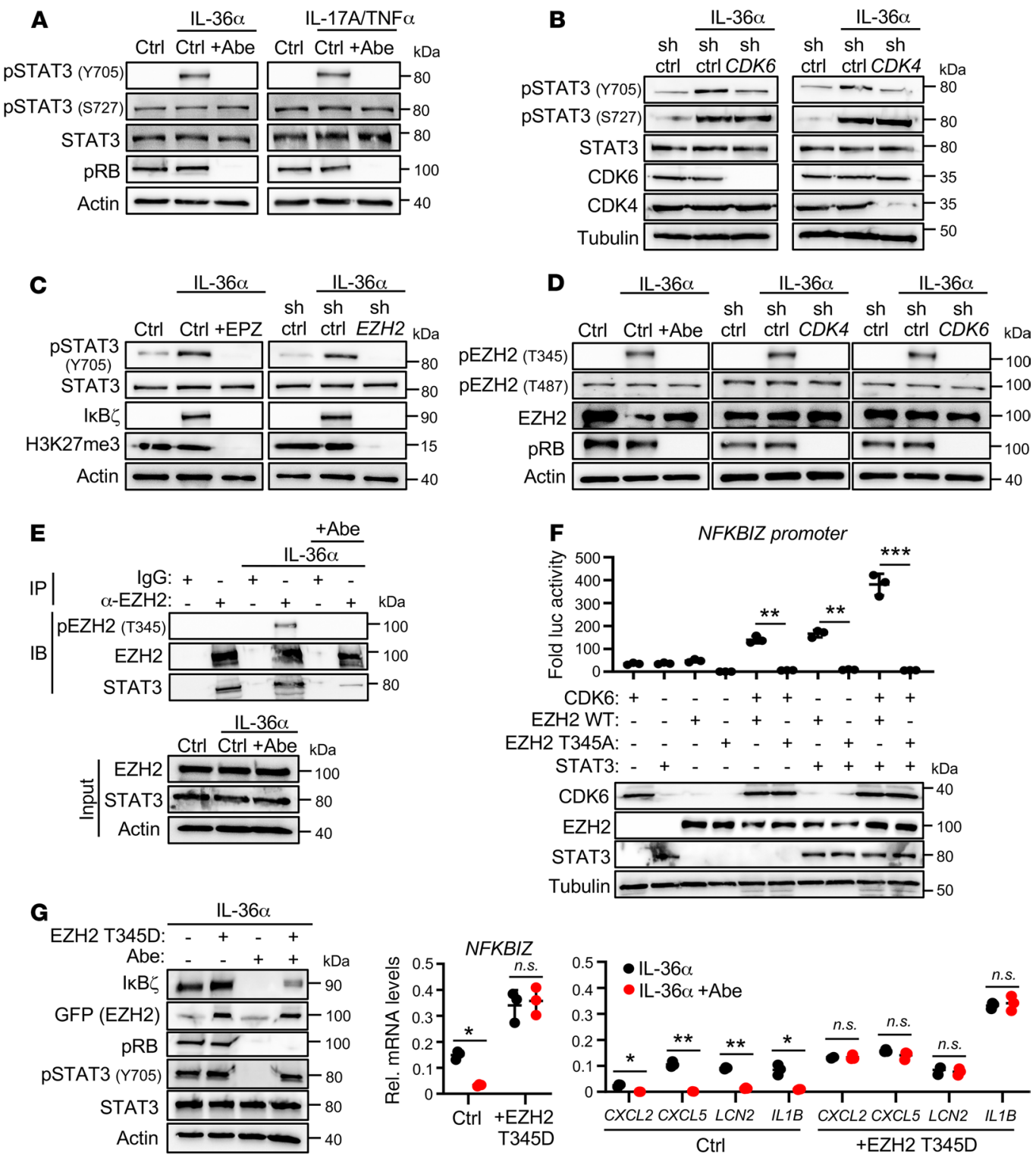

H
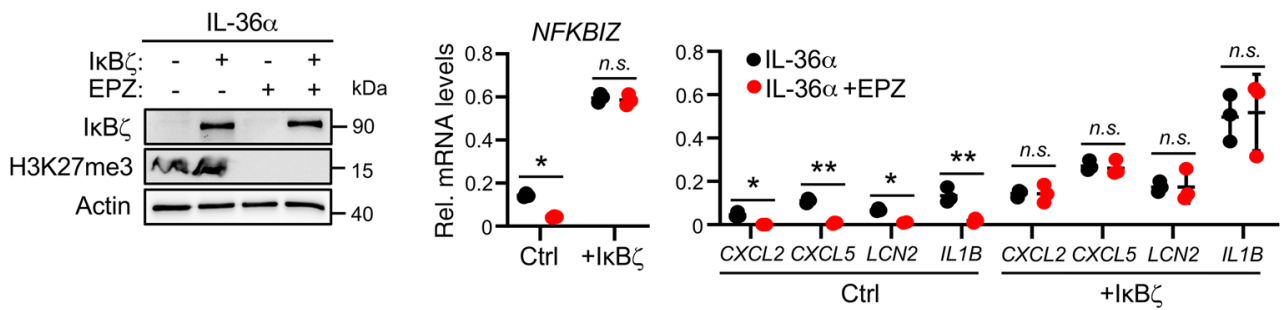

Figure 3. CDK4 and CDK6 phosphorylate EZH2 to induce STAT3 activation. (A) STAT3 activity was detected by analyzing the phosphorylation state at tyrosine 705 (Y705) and threonine 727 (T727) of STAT3 in primary human keratinocytes. After overnight starvation, cells were stimulated for 1 hour with IL-36 $\alpha$ or IL-17A/ TNF- $\alpha$ in the presence or absence of abemaciclib (Abe). (B) STAT3 activity in CDK4- and CDK6-depleted keratinocytes. Stimulation as in A. (C) Immunoblot detection of phosphorylated STAT3 (Y705) in IL-36 $\alpha$-stimulated keratinocytes, in which EZH2 function was suppressed by the EZH2 inhibitor EPZ6438 (EPZ, $10 \mu \mathrm{M}$ ) or shRNA-mediated knockdown. Detection of H3K27me3 controlled effective EZH2 inhibition or depletion. (D) Immunoblot detection of phosphorylated EZH2 at threonine 345 (T345) and threonine 487 (T487) in abemaciclib-treated or CDK4/6-depleted keratinocytes following stimulation with IL-36 $\alpha$. (E) Coimmunoprecipitation of EZH2 and STAT3 in HaCaT cells treated for 30 minutes with IL-36 $\alpha$ in the presence or absence of abemaciclib. An EZH2-specific antibody or IgC was used for pull down of protein complexes. STAT3 and pEZH2 (T345) were detected by immunoblotting. (F) Luciferase activity assay of the NFKBIZ promoter in HEK293T cells, which transiently overexpress CDK6, WT EZH2 (wt), mutant EZH2 (T345A), or STAT3, alone or in combination. Equal protein expression was detected by immunoblotting. $n=3 \pm$ SD. (C) Gene expression in IL-36 $\alpha$ - and abemaciclib-treated, primary keratinocytes following transient expression of a phospho-mimicking

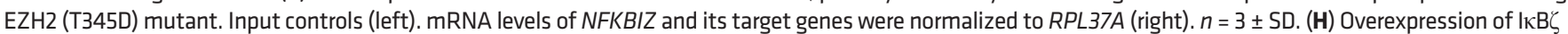
overrides the inhibitory effects of EPZ6438 (EPZ) on IL-36 $\alpha$-stimulated gene expression in primary keratinocytes. $n=3 \pm$ SD. Significance was calculated using a 1-way ANOVA for multiple groups and a 2-tailed Student's $t$ test for comparing 2 groups: ${ }^{*} P<0.05 ;{ }^{* *} P<0.01 ;{ }^{* *} P<0.001$. 
A
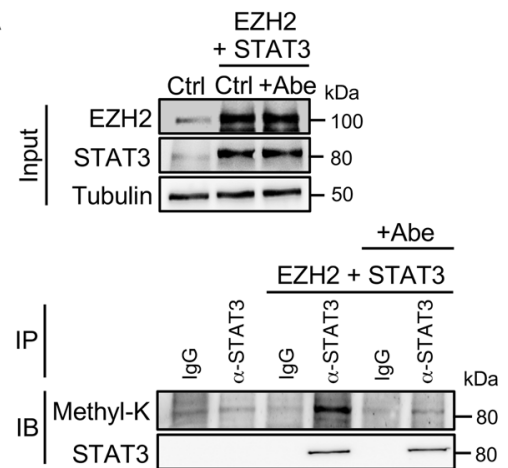

C

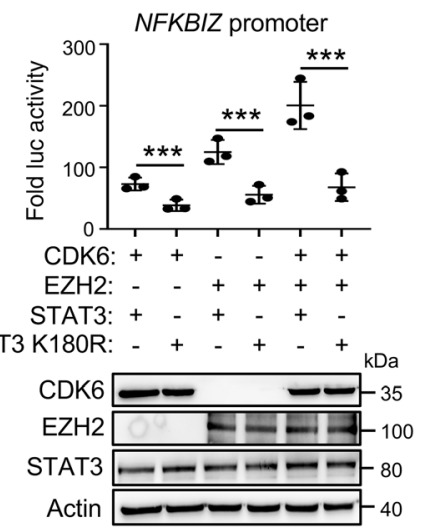

B

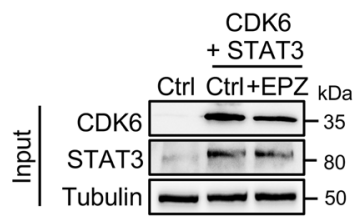

D
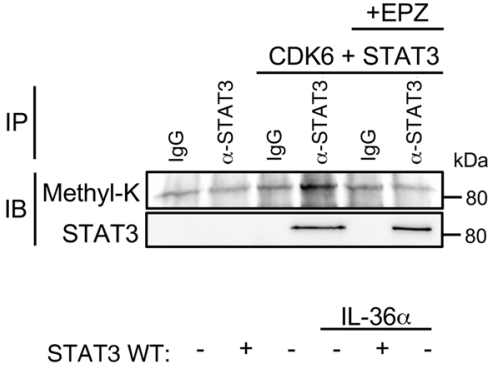

STAT3 K180R: $-\quad+\quad+\quad-\quad+k D a$

$\mathrm{IKB} \zeta \quad-$

STAT3 - - -

Actin -40

+ IL-36a
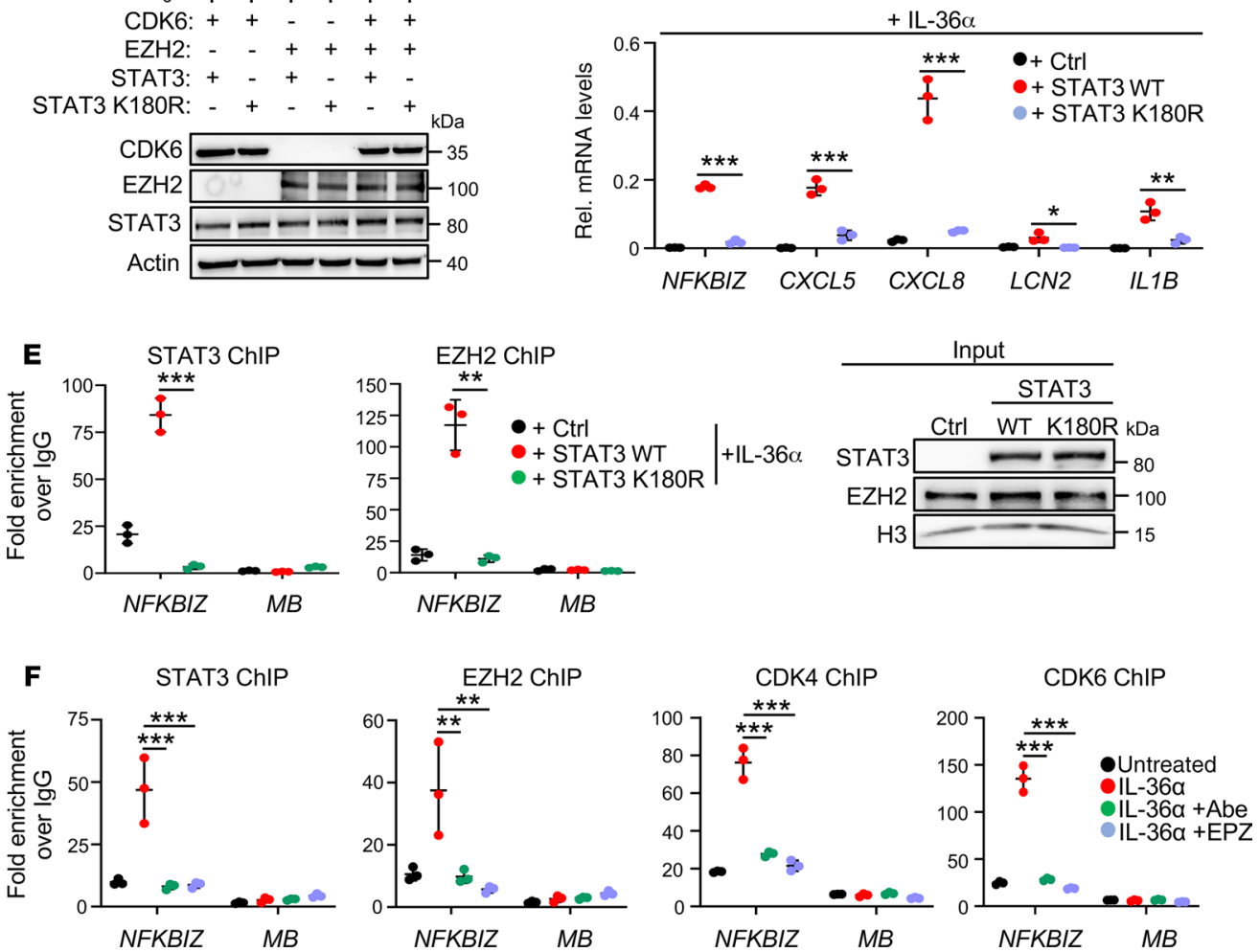

Figure 4. CDK4/6-dependent, EZH2-mediated methylation of STAT3 at lysine 180 induces IкB $\zeta$ expression in keratinocytes. (A and B) Detection of methylated STAT3 by coimmunoprecipitation. EZH2 and STAT3 (A) or CDK6 and STAT3 (B) were transiently expressed in HEK293T cells. After 1 hour of treatment with (A) abemaciclib (Abe) or (B) EPZ6438 (EPZ), cell lysates were prepared and subjected to immunoprecipitation using a STAT3-specific antibody or control IgG. (C) NFKBIZ promoter-driven luciferase activity in HEK293T cells, transiently expressing CDK6 and EZH2, alone or in combination with WT (wt) STAT3 or methylation-defective STAT3 mutant (K180R). $n=3 \pm$ SD. (D) Analysis of I $\kappa$ B $\zeta$ and I $\kappa B \zeta$ target gene expression in STAT3 wt or STAT3 K180R-expressing HaCaT cells. STAT3 wt or STAT3 K180R constructs were transiently expressed in STAT3-KO HaCaT cells, followed by stimulation for 1 hour with IL-36 $\alpha . n=3 \pm$ SD. (E) Chromatin immunoprecipitation (ChIP) of STAT3, EZH2, or IgG control in STAT3-KO HaCaT cells reconstituted with either STAT3 wt or STAT3 K180R after 30 minutes of stimulation with IL-36 $\alpha$. Fold enrichment at the NFKBIZ promoter or at the myoglobin genomic region (MB; as negative control) was calculated relative to the IgG control. $n=3 \pm$ SD. (F) ChIP of STAT3, EZH2, CDK4, and CDK6 in IL-36 $\alpha$-stimulated HaCaT cells stimulated for 30 minutes with IL-36 $\alpha$. Shown is the fold enrichment over IgG control. $n=3 \pm$ SD. Significance was calculated using a 1-way ANOVA for multiple groups and a 2-tailed Student's $t$ test for comparing 2 groups: ${ }^{*} P<0.05 ;{ }^{* *} P<0.01 ;{ }^{* *} P<0.001$

localization (34-36). Thus, we immunoprecipitated STAT3 in STAT3- and EZH2-overexpressing HEK293T cells in the presence or absence of abemaciclib, and analyzed the methylation status of STAT3 using a pan-methyl-lysine-specific antibody.
Simultaneous overexpression of EZH2 and STAT3 induced methylation of STAT3, as expected, whereas CDK4/6 inhibition abrogated lysine methylation of STAT3 (Figure 4A). Furthermore, lysine methylation of STAT3 was detectable upon 
co-overexpression of CDK6 and STAT3, whereas pharmacological EZH2 inhibition abrogated STAT3 methylation (Figure 4B). Thus, CDK4/6 might indeed trigger an EZH2-dependent methylation and activation of STAT3.

EZH2-dependent methylation sites of STAT3 at lysine 49, 140 , and 180 were previously identified by mass spectrometric analyses in tumor cells (34-36). Thus, we substituted all 3 lysine methylation sites with arginine residues and tested the STAT3 mutants for their potential to activate NFKBIZ luciferase promoter expression. Whereas mutations of STAT3 at K49 and K140 had no effect on the induction of NFKBIZ promoter expression, alone or in combination with CDK6 and EZH2 (Supplemental Figure 4A), mutation of lysine 180 (STAT3 K180R), abrogated STAT3-mediated NFKBIZ promoter activation (Figure 4C). Thus, we hypothesized that CDK4/6-activated EZH2 methylates STAT3 at lysine 180 , which is needed to induce $\mathrm{I} \kappa \mathrm{B} \zeta$ expression in stimulated keratinocytes. In agreement, reconstitution of CRISPR/Cas9-generated STAT3 knockout keratinocytes with WT STAT3, but not with

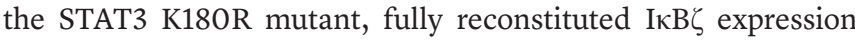
and $\mathrm{I} \kappa \mathrm{B} \zeta$-mediated target gene induction upon IL-36 $\alpha$ or IL-17A/ TNF- $\alpha$ stimulation (Figure $4 \mathrm{D}$ and Supplemental Figure $4 \mathrm{~B}$ ). This correlated with an absence of nuclear translocation of STAT3 K180R in IL-36 $\alpha$-treated keratinocytes (Supplemental Figure 4C), as observed before (34). Accordingly, mutant STAT3 K180R and EZH2 were unable to bind to the NFKBIZ promoter region in IL-36 $\alpha$-stimulated keratinocytes (Figure 4E). Thus, whereas IL-36 $\alpha$ stimulation triggered WT STAT3 binding to the NFKBIZ promoter region together with EZH2 and CDK4/6, inhibition of CDK4/6 (Abe) or EZH2 (EPZ) abrogated the recruitment of this multiprotein complex (Figure $4 \mathrm{~F}$ ). These results therefore suggest that CDK4 and CDK6 phosphorylate EZH2 to induce EZH2dependent K180 STAT3 methylation, leading to the recruitment of the heteromeric complex to the NFKBIZ promoter and subsequent induction of $\mathrm{I} \kappa \mathrm{B} \zeta$ and its target gene expression in keratinocytes.

Finally, we wanted to know if cytokines that activate the classical JAK/STAT3 pathway could override CDK4/6- or EZH2 inhibitor-mediated suppression of STAT3 activation. As revealed before (41-43), stimulation of primary keratinocytes with the cytokines IL-6, IL-20, or IL-22, which are upregulated in psoriatic lesions, led to the phosphorylation of STAT3 (Supplemental Figure 4D and refs. 41-43). Of note, neither abemaciclib nor EPZ6438 was able to abrogate STAT3 phosphorylation under these conditions, implying that CDK4/6 and EZH2 specifically control phosphorylation of STAT3 upon stimulation with IL-36 $\alpha$ or IL-17A/TNF- $\alpha$. Importantly, even though IL-6, IL-20, or IL-22 could reestablish STAT3 phosphorylation in IL-36 $\alpha$ - and abemaciclib-treated keratinocytes, stimulation with these cytokines failed to restore IкB $\zeta$ and its target gene expression (Supplemental Figure 4, E and F), nor was it able to reestablish the nuclear translocation of STAT3 in keratinocytes (Supplemental Figure 4G). This finding implies that CDK4/6-EZH2-mediated methylation of STAT3 is distinguished from the activation of STAT3 by the JAK/STAT pathway.

Human and murine psoriatic lesions are characterized by overexpression of cyclin D2, cyclin D3, and EZH2. Our findings suggest that CDK4 and CDK6 mediate the phosphorylation of EZH2 in a cyclin D-dependent manner, leading to STAT3 activation and $\mathrm{I} \kappa \mathrm{B} \zeta$ expression. We therefore investigated a potential relevance of this pathway in skin biopsies from patients with psoriasis. Human psoriatic lesions, compared with nonpsoriatic lesions or unaffected skin, were characterized by an upregulation of CCND2 and CCND3 (Figure 5A). In contrast, CCND1 levels were decreased or remained unaffected in lesional skin biopsies. This is in line with our previous observation (Supplemental Figure 2, C and D) that cyclin D1, unlike cyclin D2 and cyclin D3, did not synergize with CDK4/6 and STAT3 in increasing NFKBIZ promoter activity or expression of $\mathrm{I} \kappa \mathrm{B} \zeta$ and its target genes. In addition, EZH2 mRNA levels were significantly upregulated in human psoriatic skin lesions (Figure 5B). Immunohistochemistry further demonstrated that, on the protein level, human EZH2, which was only weakly expressed in normal skin, was strongly overexpressed in the basal cell compartment of psoriatic skin lesions, revealing a typical nuclear localization (Figure 5C).

Next, we asked if an upregulation of cyclin D2, cyclin D3, and $\mathrm{EZH} 2$ can be also detected in relevant psoriasis mouse models. In the standard model using the TLR7 agonist imiquimod (IMQ), psoriasis-like skin inflammation was triggered by daily application of an IMQ-containing cream on the ears for 6 days, while in a second model daily intradermal injections of IL-36 $\alpha$ into the skin of mouse ears were employed for 5 consecutive days. After 6 or 7 days of treatment, not only skin inflammation but also increased expression of Ccnd2, Ccnd3, and Ezh2 mRNA was detectable in both animal models (Figure 5, D and E). Moreover, increased protein levels of cyclin D2/D3 and EZH2 could be detected in the epidermis of IMQ-treated mouse ears (Figure 5F). Thus, in addition to the previously demonstrated overexpression of $\mathrm{I} \kappa \mathrm{B} \zeta$ in psoriasis $(8,9)$, a hyperactive cyclin D-CDK4/6 pathway and elevated EZH2 expression are evident in murine and human psoriatic skin lesions.

Topical application of inhibitors targeting CDK4/6 or EZH2 protects against experimental psoriasis in vivo. $\mathrm{I} \kappa \mathrm{B} \zeta$ is one of the key transcriptional regulators in the pathogenesis of psoriasis $(8,9)$. Due to our finding that CDK4/6 and EZH2 inhibitors suppressed psoriasis-related, proinflammatory gene expression downstream of IL-36 $\alpha$ or IL-17A/TNF- $\alpha$, we next investigated the potential of CDK4/6 and EZH2 inhibitors to block experimental psoriasis in vivo. Moreover, we reasoned that topical application of both inhibitors would be sufficient, as the epidermis constitutes the main target for CDK4/6 and EZH2 inhibition. A prerequisite for efficient takeup of small-molecule inhibitors from the skin are hydrophobicity of these substances. Thus, we selected more hydrophobic inhibitors, such as abemaciclib (for CDK4/6 inhibition) or CPI-169 (44) (for EZH2 inhibition) that are more likely to penetrate the outer skin barrier. Psoriasis-like skin inflammation was induced in the abovementioned psoriasis model by daily application of an IMQ-containing cream on the ears of WT mice for 6 days, before animals were sacrificed and analyzed at day 6 (45). Abemaciclib, CPI-169, and ethanol as vehicle control were applied daily on the ear skin in parallel to IMQ (Supplemental Figure 5A). Whereas IMQ-treated ears exerted ear thickening, along with keratinocyte hyperproliferation and immune cell infiltration, topical application of abemaciclib or CPI-169 strongly suppressed IMQ-induced, psoriasis-like skin inflammation (Figure 6, A and B). Both inhibitors effectively penetrated the skin and inhibited CDK4/6 or EZH2, 

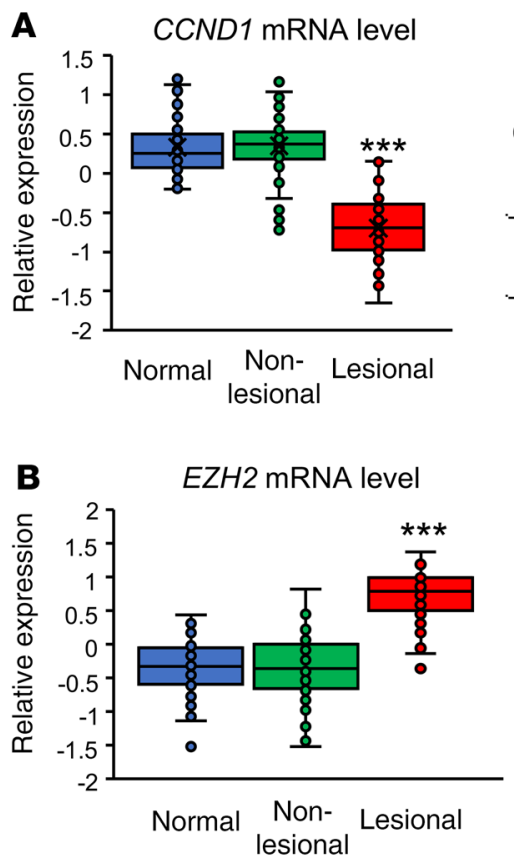
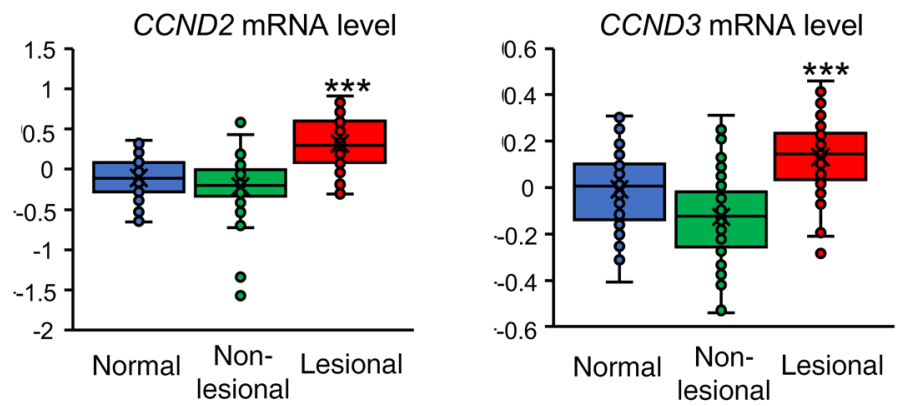
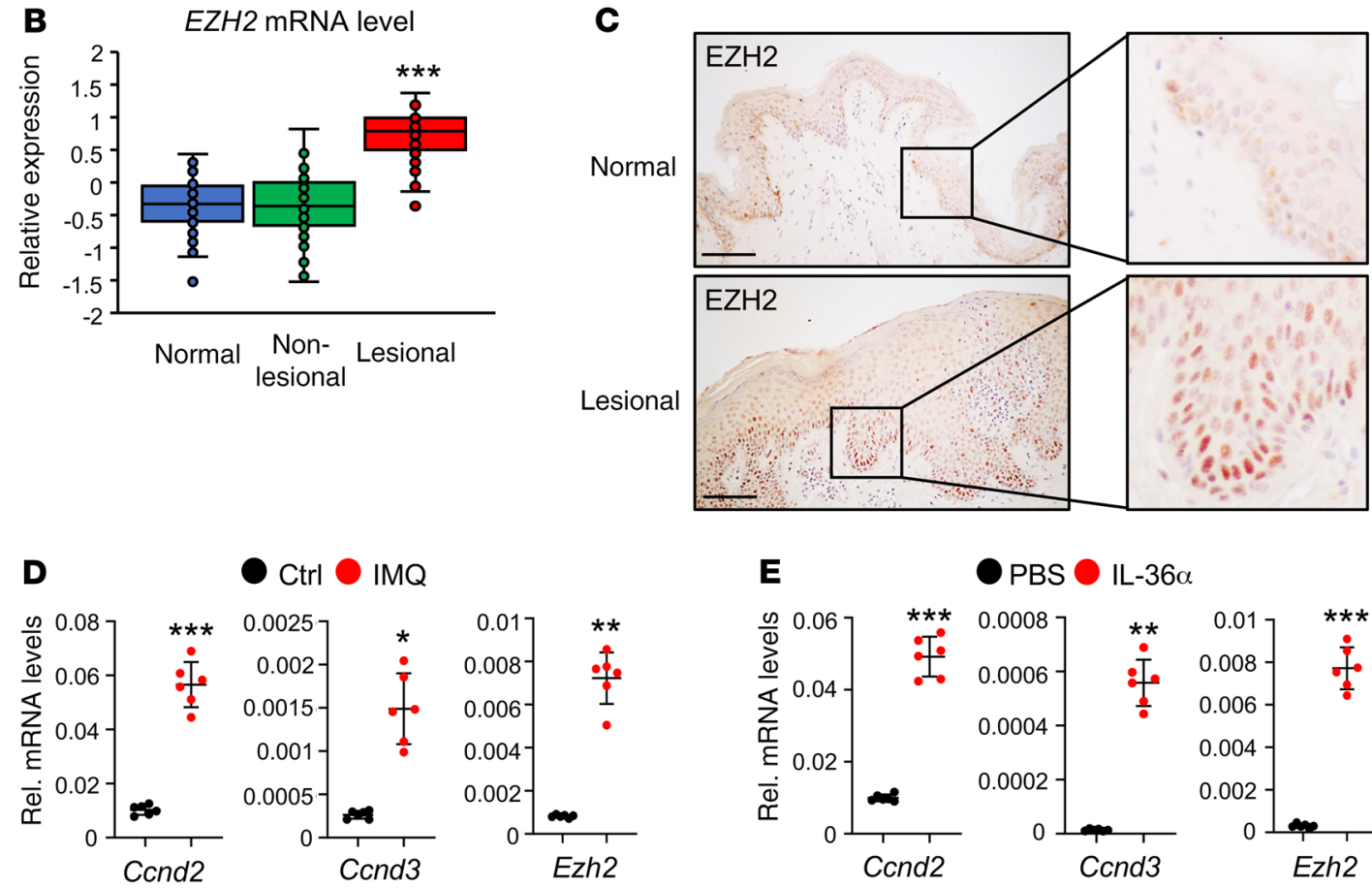

PBS IL-36 $\alpha$

$\mathbf{F}$

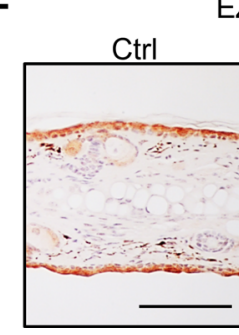

$\mathrm{EZH} 2$

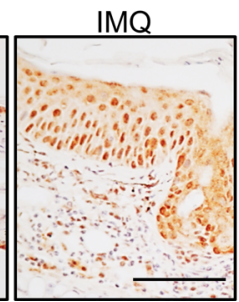

Cyclin D2

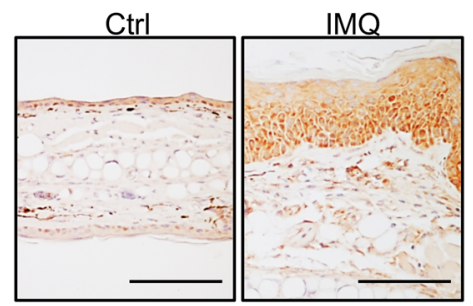

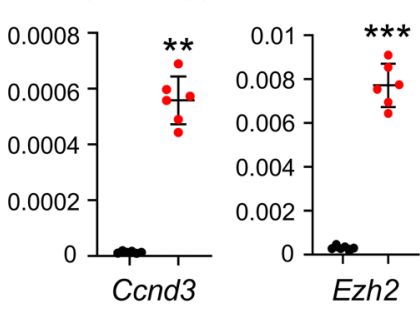

Figure 5. Increased expression of cyclin D2, cyclin D3, and EZH2 in human and murine psoriasis. (A) Expression data from skin biopsies of 64 healthy individuals and 58 patients with psoriasis were analyzed from the GEO profile data set GDS4602. Shown are normalized expression values for CCND1, CCND2, and CCND3. EZH2 mRNA (B) and protein levels (C) in human skin samples from healthy individuals and patients with psoriasis; retrieved from the same data set as in A and B. Significance was calculated with a 1-way ANOVA test: ${ }^{*} P<0.05 ;{ }^{*} P<0.01 ;{ }^{*}{ }^{*} P<0.001$. Scale bars: $100 \mu$ m. (D) Analysis of Ccnd2, Ccnd3, and Ezh2 mRNA levels in IMQ-treated mice ears at day 6. Values were normalized to Actin. $n=6$ per group \pm SEM. (E) Analysis of Ccnd2, Ccnd3, and Ezh2 mRNA levels in IL-36 $\alpha$-treated mice ears at day $5 . n=6$ per group \pm SEM. Significance was calculated using a 2-tailed Student's $t$ test: ${ }^{*} P<0.05 ;{ }^{*} P<0.01$; ${ }^{* * *} P<0.001$. (F) IHC staining of EZH2, cyclin D2, and cyclin D3 in untreated (Ctrl) and IMQ-treated mouse ears at day 6 . Scale bars: $40 \mu \mathrm{m}$.

as detected by loss of $\mathrm{pRB}$ (for CDK4/6 inhibition) or H3K27me3 (for EZH2 inhibition) expression in the epidermis of treated mice (Figure 6C). Moreover, abemaciclib treatment significantly suppressed the infiltration of neutrophils, macrophages, and $\mathrm{T}$ cells in IMQ-treated mice (Figure 6D), while topical application of the EZH2 inhibitor CPI-169 fully abrogated immune cell infiltration upon IMQ treatment (Figure 6E). Of note, also the number of infiltrating plasmacytoid dendritic cells (pDCs) and myeloid dendritic cells (mDCs) was significantly suppressed by application of both inhibitors (Supplemental Figure 5B). Importantly, whereas IMQ treatment effectively induced IкB $\zeta$ expression in the skin, along with phosphorylation of EZH2 at T345 
A
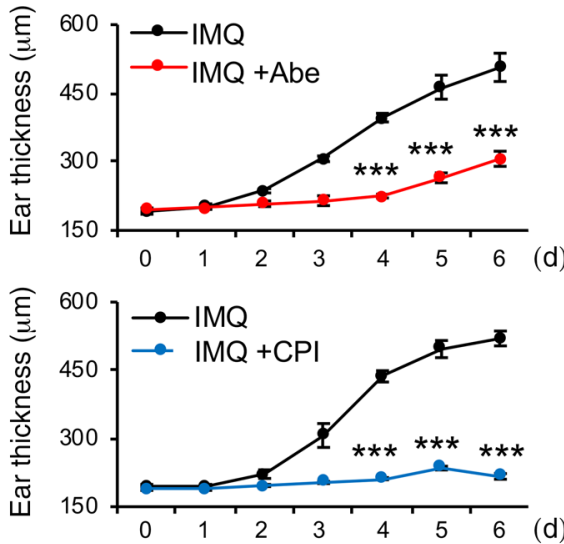

\section{C}
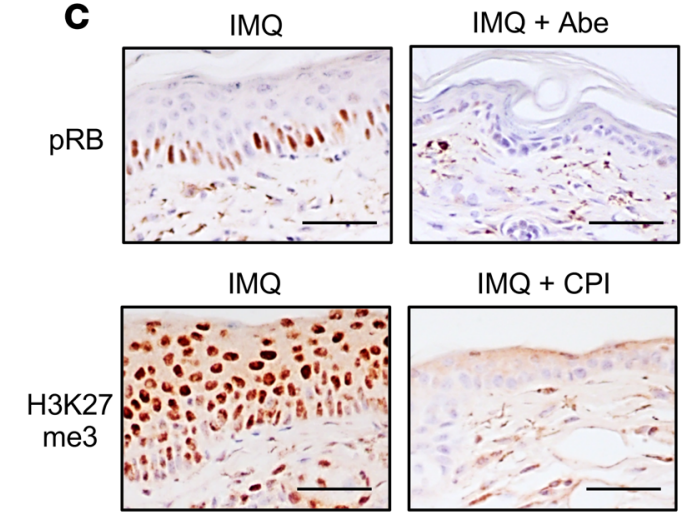

$|\mathrm{MQ}+\mathrm{CP}|$
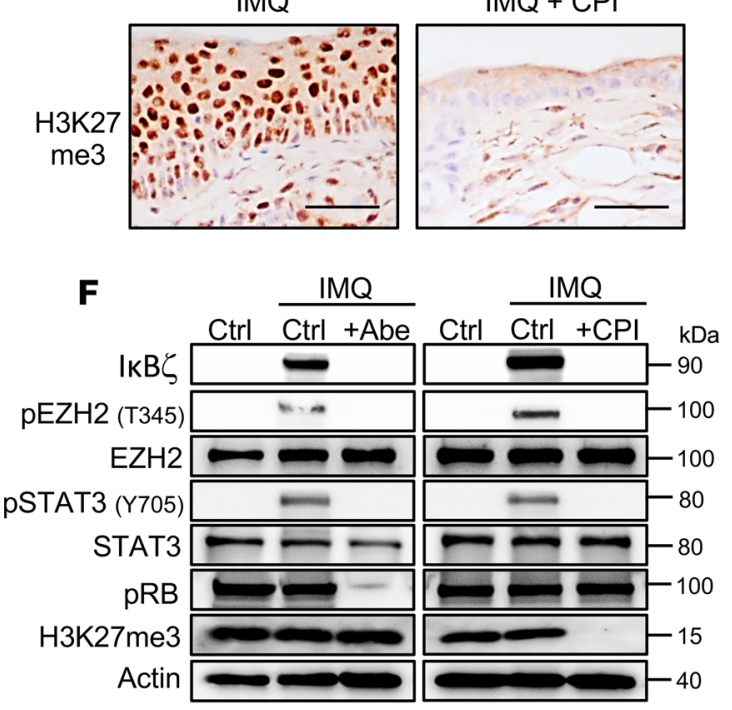

H

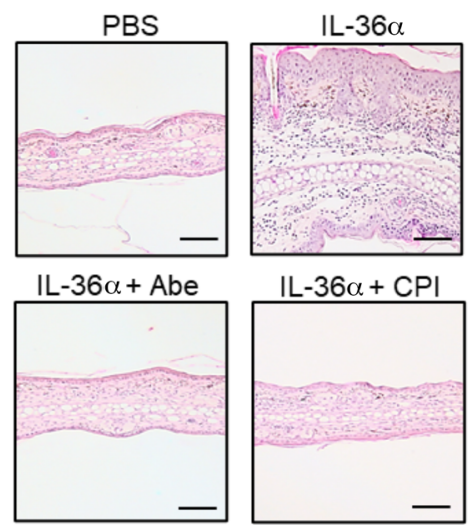

B

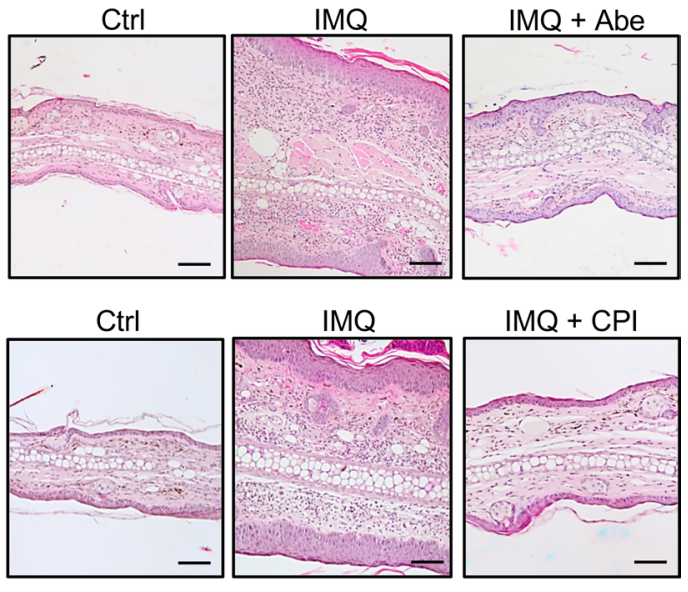

D
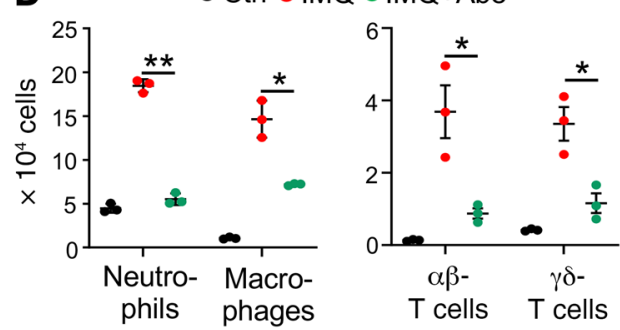

E
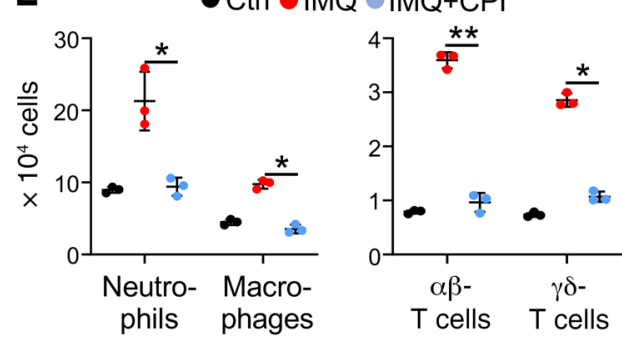

G
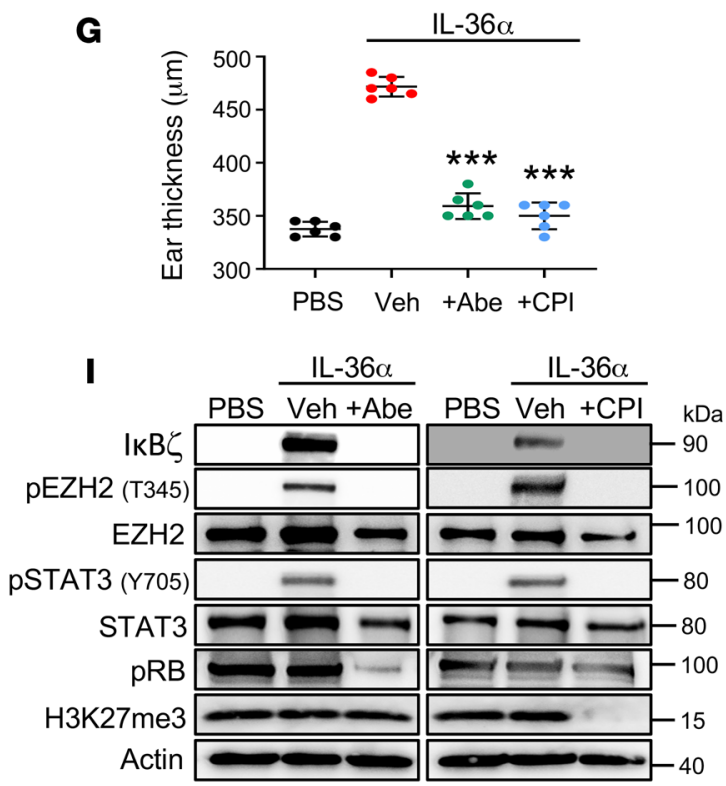
Figure 6. CDK4/6 and EZH2 inhibition prevents IMQ- and IL-36-mediated psoriasis-like skin lesions in vivo. (A) Ear thickness measurements during topical treatment of mice with IMQ with or without abemaciclib (Abe; $10 \mu \mathrm{L}$ of a $2 \%$ solution) or the EZH2 inhibitor CPI-169 (CPI, $10 \mu \mathrm{L}$ of a $5 \%$ solution). $n=6$ mice per group \pm SEM. (B) H\&E staining of untreated (Ctrl), IMQ-, IMQ and Abe-, or IMQ and CPI-treated ears. Scale bars: $100 \mu \mathrm{m}$. (C) Phospho-RB (pRB) and H3K27me3 staining after 6 days of treatment validated effective CDK4/6 and EZH2 inhibition, respectively. Scale bars: $40 \mu \mathrm{m}$. (D) Infiltrating immune cells in mouse ears at day 6 of treatment were quantified as follows: Neutrophils: CD45+, CD11b+, Ly6G+; macrophages: CD45+, CD11b+, F4 $/ 80^{+}$; T cells: $\mathrm{CD}_{4} 5^{+}, \mathrm{CD}^{+}$, and $\alpha \beta-\mathrm{TCR}^{+}$or $\gamma \delta-\mathrm{TCR}^{+} . n=3$ mice per group \pm SEM. (E) Flow cytometry analysis of IMQ-treated or IMQ and CPI-169-treated mouse ears at day 6. (F) Protein levels in untreated (Ctrl) and treated mouse skin tissue at day 6. (G) Ear thickness of IL-36 $\alpha$-treated mice at day 5. Ears of mice were daily treated by intradermal injections with $1 \mu \mathrm{g} \mathrm{IL-} 36 \alpha$. Control mice received injections with PBS. Additionally, mice received topical treatment with ethanol as control (Veh), $\%$ abemaciclib (Abe), or $5 \%$ CPI-169 (CPI). $n=6$ mice per group \pm SEM. (H) H\&E staining of PBS- or IL-36 $\alpha$-treated ears at day 5. Scale bars: $100 \mu \mathrm{m}$. (I) Immunoblot analysis of I KB, EZH2 phosphorylation (pEZH2 T345) and STAT3 activation (pSTAT3 Y705) in treated mouse skin tissue at day 5. pRB and H3K27me3 were analyzed as positive controls for drug action. Significance was calculated using a 1-way ANOVA for multiple groups and a 2-tailed Student's $t$ test for comparing 2 groups: ${ }^{*} P<0.05 ;{ }^{*} P<0.01 ;{ }^{* *} P<0.001$.

and of STAT3 at Y705, topical administration of abemaciclib or CPI-169 completely abrogated these signaling events (Figure 6F and Supplemental Figure 5C). As a positive control, stabilization of the CDK4/6 substrate pRB (46) and EZH2-directed H3K27 methylation were strongly reduced in either CDK4/6 or EZH2 inhibitor-treated mouse skin (Figure 6F). Accordingly, expression of I $\kappa$ B $\zeta$ target genes, such as $C x c l 2$ and $C x c l 5$, and DC- and T cell-derived cytokines, such as $I l 17 a$ or $I l 23 a$, was significantly downregulated in IMQ- and abemaciclib- or IMQ- and CPI-169treated skin (Supplemental Figure 5D).

Treatment of mice with the TLR7 agonist IMQ represents a standard mouse model for psoriasis (45). However, IMQ activates immune cells in the first instance, rather than an initial keratinocyte-derived proinflammatory response, as it is likely to happen in human psoriasis pathogenesis. Thus, we additionally investigated the therapeutic effects of abemaciclib or CPI-169 in an IL-36-triggered psoriasis-like dermatitis mouse model (Supplemental Figure 5E). As previously reported (9, 47 ), repeated intradermal injections of IL-36 $\alpha$ into the skin of mouse ears induced ear swelling, and keratinocyte hyperproliferation along with immune cell infiltration (Figure 6, G and $\mathrm{H})$. As a control for drug penetration in the skin of IL-36-treated animals, effective inhibition of CDK4/6 and EZH2 methyltransferase activity was controlled by staining for $\mathrm{pRB}$ and H3K27me3, respectively (Supplemental Figure 5F). Similar to the IMQ mouse model, topical application of abemaciclib or CPI-169 effectively blocked keratinocyte hyperproliferation and immune cell infiltration (Figure 6, G and H). Moreover, both inhibitors suppressed IL-36-mediated expression of I $\mathrm{B} \zeta$, phosphorylation of EZH2 at T345, and activation of STAT3 (pSTAT3 Y705) in the skin of treated mouse ears (Figure 6I). Accordingly, I $\mathrm{I} \mathrm{B} \zeta$ target gene expression and key cytokine expression, such as $I l 17 a$ and Il23a, were effectively blocked as well (Supplemental Figure 5G). Thus, inhibition of CDK4/6 or
EZH2 in IMQ- or IL-36-mediated psoriasis-like skin inflammation mouse models effectively prevented psoriasis induction in vivo, by suppressing STAT3-mediated induction of IкB $\zeta$ expression and $\mathrm{I} \kappa \mathrm{B} \zeta$ target gene expression.

CDK4/6 and EZH2 inhibitors effectively attenuate already established psoriasis-like skin inflammation in vivo. As CDK $4 / 6$ and EZH2 inhibitors could fully prevent the onset of psoriasis in vivo, we next investigated if inhibition of the CDK4/6-EZH2 pathway also attenuates already established psoriatic disease. We therefore first induced psoriasis-like skin inflammation in mice with IMQ and then topically applied abemaciclib or CPI-169 after day 2 of IMQ treatment (Figure 7A). Already, 3 treatments with IMQ effectively induced ear swelling and keratinocyte hyperproliferation. These psoriasis-like symptoms could be fully reversed by starting topical application of abemaciclib or CPI-169 (Figure 7, B and C, and Supplemental Figure 6A). Moreover, psoriasisrelated, proinflammatory gene expression as well as infiltration of neutrophils, macrophages, and T cells, which was detectable at the third day of IMQ treatment, were fully resolved by both inhibitors (Supplemental Figure 6B and Figure 7D). Finally, inhibition of CDK4/6 or EZH2 abrogated I $\mathrm{EB} \zeta$ expression as well as phosphorylation of STAT3, as detected by immunoblot analyses of whole skin lysates at day 6 (Figure 7E).

Similar results were obtained by topical application of abemaciclib or CPI-169 on established skin lesions in the IL-36 $\alpha$ psoriasis-like mouse model. In this experimental setup, application of both inhibitors at day 4 effectively resolved IL-36 $\alpha$-induced ear swelling, keratinocyte hyperproliferation, and immune cell infiltration as well as psoriasis-associated gene expression, I $\kappa$ B $\zeta$ expression, and activation of STAT3 (Figure 7, F-I, and Supplemental Figure $6 \mathrm{C}$ ). Thus, topical application of CDK4/6 and EZH2 inhibitors not only prevented the onset of experimental skin inflammation, but also resolved already established psoriasis-associated symptoms in IMQ- or IL-36 $\alpha$-treated mice. In view of its increased activity in human psoriatic skin and the results obtained in psoriasis-like mouse models, inhibition of this pathway by topical application of CDK4/6 and EZH2 inhibitors could therefore provide a new therapeutic strategy for the treatment of patients with psoriasis.

\section{Discussion}

CDK4/6 inhibitors have been developed and approved for the treatment of patients with cancer in order to restrain hyperproliferation of tumor cells (17). Recently, it was found that CDK4 and CDK6 do not only control cell cycle progression by phosphorylation of $\mathrm{RB}$, but also regulate immune cell differentiation and function $(20,21,48)$. In this context, CDK4 and CDK6 have been implicated as transcriptional cofactors that activate a subset of $\mathrm{NF}-\kappa \mathrm{B}$ or STAT3 target genes (23-25). Based on our results in cultured keratinocytes, human skin biopsies, and mouse models, we propose to repurpose CDK4/6 inhibitors for psoriasis therapy. Moreover, our results uncovered a new pathway involving CDK4/6-mediated phosphorylation of EZH2 and EZH2-dependent methylation and activation of STAT3, leading to the inducible expression of IкB $\zeta$ and $\mathrm{I} \kappa \mathrm{B} \zeta$-dependent target genes in keratinocytes. These findings also suggest the use of EZH2 inhibitors to treat psoriasis.

I $\kappa \mathrm{B} \zeta$, encoded by $N F K B I Z$, constitutes a risk gene for the development of psoriasis (49). Moreover, we recently reported 
A

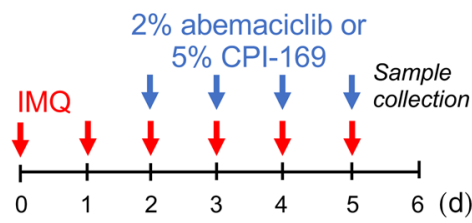

B

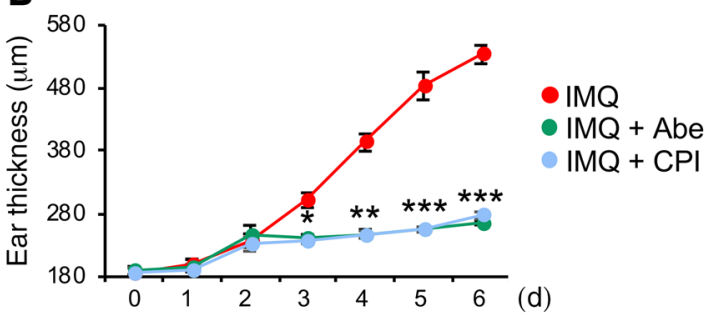

C

Ctrl

IMQ (Day 2)
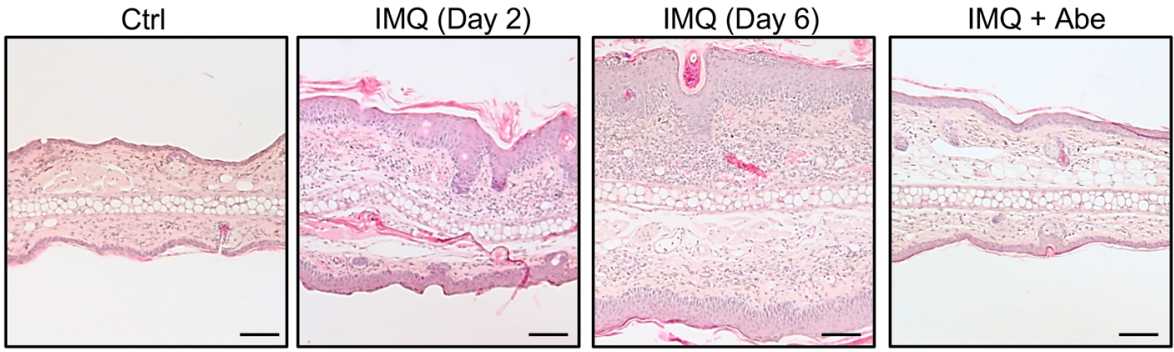

$|\mathrm{MQ}+\mathrm{CP}|$

D

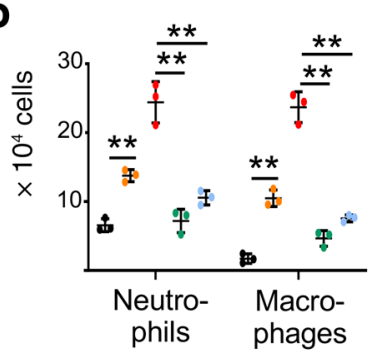

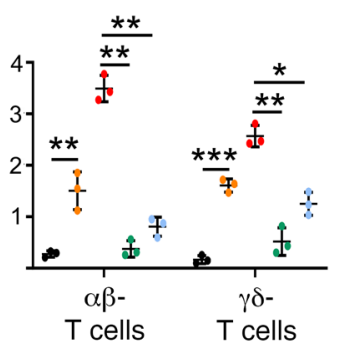

- Ctrl IIMQ - IMQ -IMQ+Abe $I \mathrm{MQ}+\mathrm{CPI}$ (Day 2) (Day 6)

$\mathbf{F}$

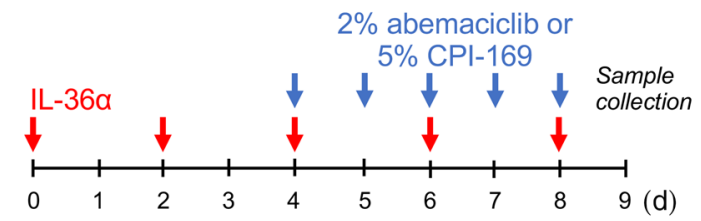

H

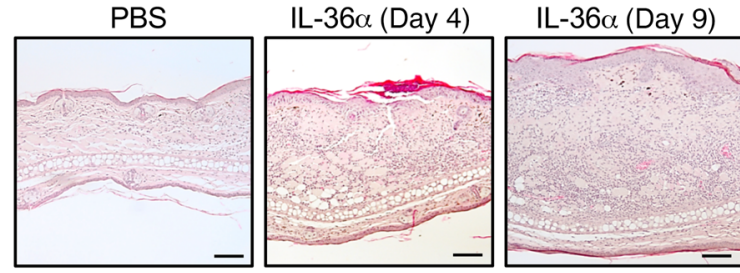

L-36 $\alpha+\mathrm{CPI}$

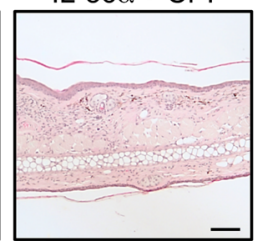

E

IMQ

$\mathrm{IMQ}$
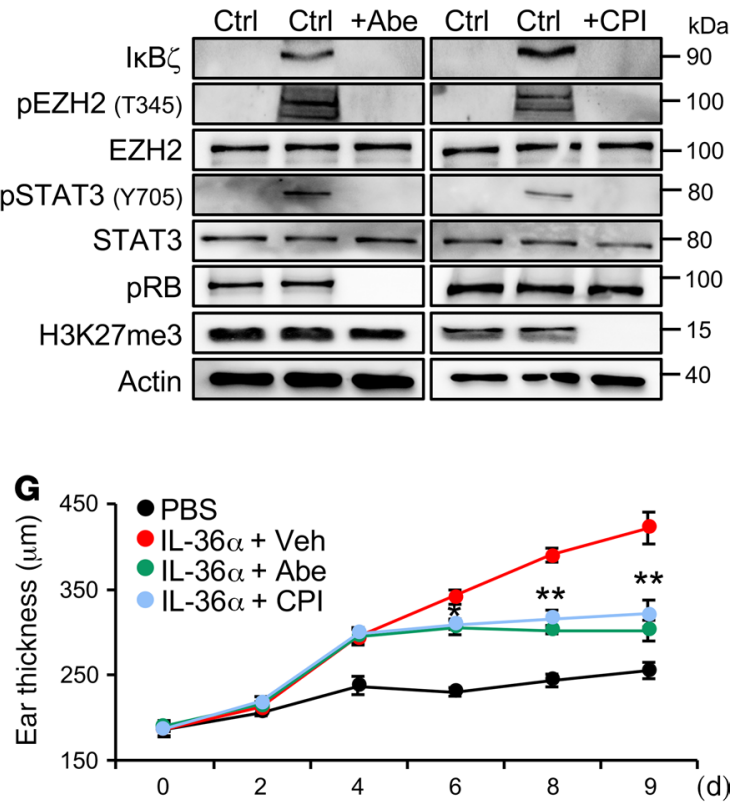

I

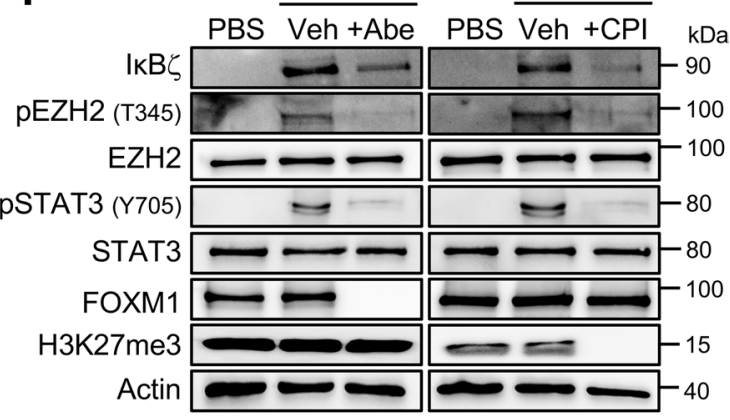


Figure 7. CDK4/6 and EZH2 inhibitors attenuate established psoriasis-like skin lesions in vivo. All analyses were performed with $n=6$ mice per group \pm SEM. (A) Treatment scheme for the therapy using the IMQ mouse model. To explore whether CDK4/6 and EZH2 inhibitors suppress already-established psoriasis-like skin inflammation, mice were first treated with IMQ, followed by the application of 2\% abemaciclib or 5\% CPI-169 solution starting at the third IMQ application. (B) Ear thickness measurements during treatment. (C) H\&E staining of untreated (Ctrl), IMQ-, IMQ and Abe-, or IMQ and CPI-treated ears. H\&E staining shows the prevalence of psoriasis-like symptoms at IMQ day 2 when the inhibitors were applied for the first time. Scale bars: 100 $\mu \mathrm{m}$. (D) Quantification of infiltrating immune cells in mouse ears at day 6. Immune cell subpopulations were quantified as in Figure 6D. $n=3$ mice per group \pm SEM. (E) Protein levels in untreated (Ctrl) and IMQ-treated mouse skin tissue in the presence or absence of abemaciclib or CPI-169 at day 6 . Mice were treated as in A. FOXM1 and H3K27me3 were analyzed as positive controls for drug action. (F) Treatment scheme in the IL-36-induced psoriasis mouse model. IL-36-mediated psoriasis-like dermatitis was induced by administration of $1 \mu \mathrm{g} \mathrm{IL}-36 \alpha$ at every second day. Control mice received PBS. Starting from day 4 of IL-36 $\alpha$ injection, ethanol as Vehicle (Veh), $2 \%$ abemaciclib, or 5\% CPI-169 were daily applied by topical administration. (C) Ear thickness measurements during IL-36 $\alpha$ treatment. (H) H\&E staining of PBSor IL-36 $\alpha$-treated ears at day 9. Scale bars: $100 \mu \mathrm{m}$. (I) Immunoblot analysis in IL-36 $\alpha$-treated mouse skin tissue at day 9 . Significance was calculated using a 1-way ANOVA for multiple groups and a 2-tailed Student's $t$ test for comparing 2 groups: ${ }^{*} P<0.05 ;{ }^{* *} P<0.01 ;{ }^{* *} P<0.001$.

that $\mathrm{I} \kappa \mathrm{B} \zeta$ is overexpressed in human psoriatic lesions, whereas global and keratinocyte-specific I $\kappa \mathrm{B} \zeta \mathrm{KO}$ mice are completely protected against psoriasis-like skin inflammation in several psoriasis models $(8,9,50)$. Mechanistically, $\mathrm{I} \kappa \mathrm{B} \zeta$ is transcriptionally induced in keratinocytes by IL-17 and IL-36, which triggers the expression of psoriasis-relevant target genes encoding for selective chemokines and cytokines and antimicrobial proteins. Deficiency of $\mathrm{I} \kappa \mathrm{B} \zeta$ therefore prevents the recruitment of neutrophils and monocytes that are needed for skin inflammation $(9,50)$. Collectively, our data suggest that interfering with I $\kappa \zeta$ $\zeta$ expression or function in keratinocytes might be a promising strategy for psoriasis therapy. As $\mathrm{I} \kappa \mathrm{B} \zeta$ is crucial for both IL-36 and IL-17 signaling, CDK4/6 inhibitors might be applicable for different subtypes of psoriasis.

Unfortunately, based on a lack of enzyme activity, direct pharmacological inhibition of I $\mathrm{I} B \zeta$ function remains difficult (13). We therefore sought to block the transcriptional induction of $\mathrm{I} \kappa \mathrm{B} \zeta$ and identified small-molecule inhibitors

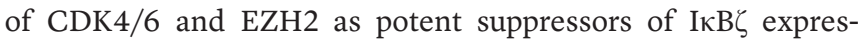
sion in keratinocytes. CDK4 and CDK6 have been previously shown to modulate several immune-relevant transcription factors by both kinase-dependent and -independent mechanisms (23-25). In the present study, we clearly demonstrate that STAT3-mediated induction of $\mathrm{I} \kappa \mathrm{B} \zeta$ expression is kinasedependent, as ATP-competitive CDK4/6 inhibitors such as abemaciclib or palbociclib abolished I $\mathrm{I} \mathrm{B} \zeta$ expression. Consistent with these findings, a hyperactive but not a dominant-negative version of CDK6 increased NFKBIZ promoter activity. Moreover, cyclin D2 and cyclin D3 elevated the expression of NFKBIZ and its target genes, supporting the need for CDK4/6 kinase activity.

Despite the requirement of the kinase activity, the involvement of CDK4/6 could be separated from its classical role in cell cycle regulation and phosphorylation of RB. Thus, depletion of
$\mathrm{RB}$ failed to restore $\mathrm{I} \kappa \mathrm{B} \zeta$ expression upon $\mathrm{CDK} 4 / 6$ inhibition. Moreover, I $\kappa \mathrm{B} \zeta$ expression was principally induced by IL-36 stimulation in all phases of the cell cycle, except for $G_{0}$-arrested cells that revealed a weaker $\mathrm{I} \kappa \mathrm{B} \zeta$ expression. Importantly, although $\mathrm{I} \kappa \mathrm{B} \zeta$ expression does not rely on CDK4/6-mediated cell cycle progression, CDK4/6 inhibitors might also have beneficial effects in psoriasis treatment by additionally blocking keratinocyte hyperproliferation, which is a hallmark of psoriasis (2).

In this study, we demonstrate a major role for STAT3 in driving keratinocyte-specific I $\mathrm{B} \zeta$ expression. I $\mathrm{\kappa} \mathrm{B} \zeta$ expression in keratinocytes is predominantly controlled from the proximal promoter 2 of the NFKBIZ locus, containing different transcription factor binding sites than the better investigated distal promoter 1 (9). So far, we have not compared the promoter usage in distinct cell types, but we consider it likely that the contribution of the individual promoters and STAT3 to I $\kappa$ B $\zeta$ expression differs among different cell types. Our experiments show that CDK4 and CDK6 do not directly phosphorylate STAT3 but EZH2, which induces $\mathrm{I} \kappa \mathrm{B} \zeta$ and $\mathrm{I} \kappa \mathrm{B} \zeta$-dependent proinflammatory target gene expression in a STAT3-dependent manner. This finding seems surprising at the first glance, since EZH2, as part of the PRC2 complex, is mainly involved in gene repression through trimethylation of H3K27. Recently, however, EZH2 was also found to induce gene expression independently of the PRC2 complex, via interaction with $\beta$-catenin or the SWI/SNF complex $(51,52)$. CDK $4 / 6$ phosphorylated EZH2 at T345, thereby inducing an EZH2-dependent methylation of STAT3 at K180, and subsequent induction of I $\kappa$ B $\zeta$ expression by STAT3. EZH2 phosphorylation at T345 was previously described to be mediated by CDK1 and CDK2, leading to an EZH2-directed epigenetic silencing of genes during G2 phase $(39,53)$. Thus, even though CDK-mediated phosphorylation of EZH2 at T345 seems to be conserved, its impact on EZH2 function and the choice of methylation substrates might depend on the specific stimulus or cell cycle phase.

Upon CDK4/6-mediated phosphorylation, EZH2 preferentially interacted with STAT3, resulting in STAT3 K180 methylation and enhanced STAT3 activation. Similar observations were made in glioblastoma, where IL-6-induced STAT3 activation is controlled by EZH2-mediated trimethylation of STAT3 at K180 (34). Thus, phosphorylation of EZH2 might induce a switch in EZH2 function from H3K27 trimethylation and transcriptional repression to noncanonical functions, including STAT3 methylation and gene activation. Whether this gene-activating function of EZH2 requires the PRC2 repressor complex or whether it is PRC2-independent remains to be resolved. In addition to its main function in transcriptional repression, non-PRC functions of EZH2 via direct binding to transcriptional regulators have been reported before. For instance, EZH2 was shown to act as a cofactor for transcription factors (such as the androgen receptor, $\beta$-catenin, or NF- $\kappa \mathrm{B})$, leading to target gene activation $(52,54,55)$. Similar to other nonhistone targets, however, the exact molecular events that link STAT3 methylation to STAT3 activation are currently unknown. In agreement with a previous report (34), our data imply that K180 methylation of STAT3 might be needed for the nuclear import of phosphorylated STAT3.

Regardless of the detailed mechanism of EZH2-mediated STAT3 activation, our study has also important clinical implications. Our 
results suggest that targeting of the CDK4/6-EZH2-STAT3 pathway not only suppresses cytokine-mediated induction of I $\kappa \mathrm{B} \zeta$ and proinflammatory target gene expression, but also inhibits immune cell recruitment and skin inflammation. We demonstrate in the IMQand IL-36-mediated psoriasis-like mouse models that both CDK4/6 and EZH2 inhibitors completely blocked the development of psoriatic skin lesions. The therapeutic effect of the inhibitors concurred with a suppression of $\mathrm{I} \kappa \mathrm{B} \zeta$ expression and a strong inhibition of $\mathrm{I} \kappa \mathrm{B} \zeta$ target gene expression, including chemokines (e.g., Cxcl2, Cxcl5), cytokines (e.g., Il1f9, Illb, Il17a, Il23a), and antimicrobial proteins

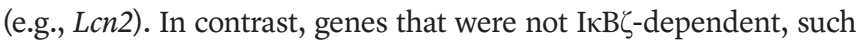
as NFKBIA and TNF, remained unaffected upon CDK4/6 or EZH2 inhibition. These findings further support the view of a rather selective role of $\mathrm{I} \kappa \mathrm{B} \zeta$ in the control of immune responses and also indicate that inhibition of $\mathrm{I} \kappa \mathrm{B} \zeta$ will be associated with fewer side effects than a broad inhibition of NF- $\mathrm{KB}$ by toxic IKK inhibitors.

In line with previous reports showing an upregulated expression of $I \kappa B \zeta$ in psoriasis $(8,9)$, we detected an increased nuclear accumulation of EZH2 and elevated cyclin D2 and D3 levels, both in mouse models of psoriasis and in human psoriatic skin lesions. Previous studies also found that mutations in the STAT3 signaling pathway constitute a risk factor for the development of psoriasis (43), while constitutively active STAT3 characterizes the epidermis of human psoriatic lesions (56). Collectively, this suggests that the CDK4/6-EZH2-STAT3 pathway is hyperactive in psoriatic skin lesions. As inhibition of $\mathrm{I} \kappa \mathrm{B} \zeta$ blocks multiple signaling pathways in psoriasis, targeting $\mathrm{I} \kappa \mathrm{B} \zeta$ might increase overall therapy responses as well as prevent the development of therapy resistance. Due to the clinical availability of hydrophobic CDK4/6 and EZH2 inhibitors, we propose formulation of these inhibitors in, for example, a cream for topical treatment of psoriatic skin lesions. Topical drug administration will also restrict potential side effects and might be especially promising for those patients who have developed resistance to current psoriasis therapies.

\section{Methods}

Cell culture and treatment. HaCaT cells were obtained from Petra Boukamp (57) and maintained in DMEM with 10\% FCS and antibiotics. Human primary keratinocytes were freshly isolated from foreskin and maintained in CnT-07S medium with gentamycin (CELLnTEC). Recombinant human IL-36 $\alpha$ (6995-IL; aa 6-158), IL-36 $\gamma$ (6835-IL; aa 18-169), and mouse IL-36 (7059-ML; aa 6-160) were purchased from R\&D Systems. Recombinant IL-17A (catalog 11340174), TNF- $\alpha$ (catalog 11343013), IL-1 $\beta$ (catalog 11340013), IL-6 (catalog 11340064), IL-20 (catalog 11340203), and IL-22 (catalog 11340223) were ordered from Immunotools. Flagellin (vacfla) and polyI:C (vac-pic) were purchased from Invivogen. In cell culture experiments, all cytokines were used at $100 \mathrm{ng} / \mathrm{mL}$ end concentration, except for IL-17A (200 ng/mL) and TNF- $\alpha$ (10 ng/ $\mathrm{mL}$ ). Flagellin was applied at $10 \mathrm{ng} / \mathrm{mL}$ and poly I:C was added at a final concentration of $100 \mathrm{ng} / \mathrm{mL}$. The following inhibitors were purchased from Selleckchem: abemaciclib mesylate (LY2835219, S17158), palbociclib isethionate (S1579), EPZ6438 (tazemetostat, S7128), and CPI-169 (S7616). If not otherwise indicated, the inhibitors were used in cell cultures at the following concentrations: abemaciclib $(16 \mu \mathrm{M})$, palbociclib $(50 \mu \mathrm{M})$, and EPZ6438 $(10 \mu \mathrm{M})$. When indicated, cells were starved overnight, before cytokine treatment, by removing cell culture supplements from the growth medium.

Generation of knockdown cells. Lentiviral particles were produced in HEK293T cells using the second-generation packaging system (pMD2.G, 12259; and psPAX2, 12260; Addgene). Keratinocytes were transduced in the presence of $8 \mu \mathrm{g} / \mathrm{mL}$ polybrene, packaging plasmids, and $5 \mu \mathrm{g}$ of the respective shRNA construct (all from Dharmacon): pLKO.1-puro (sh ctrl); pLKO.1-TRCNO000009876 (shCDK4); pLKO.1TRCNO000010473 (shCDK6);pTRIPZ-EZH2 (V2THS63066, shEZH2); pLKO.1-TRCNO000040167 (shRB); pTRIPZ noncoding ctrl (RHS4743); pLKO.1-TRCNOOOOO20840 (shSTAT3); pLKO.1-TRCNOOOO014683 (shRELA), followed by puromycin selection (1 ng/mL, Invitrogen). For induction of EZH2 knockdown, pTRIPZ ctrl and pTRIPZ-EZH2-expressing cells were treated for 24 hours with $2 \mu \mathrm{g} / \mathrm{mL}$ doxycycline (AppliChem) before stimulation and harvest of the cells.

Luciferase constructs and reporter assays. Luciferase constructs were generated as described and based on the pInducer20 plasmid (Addgene, 44012) (9). HEK293T cells $\left(1 \times 10^{4}\right)$ were transfected for 24 hours using $\mathrm{HeBS}$ buffer and $\mathrm{CaCl}_{2}$ and a mixture of $400 \mathrm{ng}$ firefly luciferase vector and $100 \mathrm{ng}$ TK-Renilla vector. For expression of other proteins, the following concentrations were purchased from Addgene: 70 ng p65 (catalog 106453), 200 ng cJun (catalog 102758), STAT3 (catalog 8706) or EZH2-HA (catalog 24230) constructs, and $500 \mathrm{ng}$ CDK4-HA (catalog 1868), CDK6-HA (catalog 1866), CDK6DN (catalog 1869), cyclin D1-HA (catalog 11181), cyclin D2 (catalog 8958), and cyclin D3 (catalog 10912). Additionally, CDK6 S178P expression construct was a gift from Michael Kracht (University of Giessen, Germany) (58). For transfection of HaCaT cells, $3 \times 10^{5}$ cells were transfected for 4 hours using Lipofectamine 3000 and a mixture of 800 ng firefly luciferase vector, $200 \mathrm{ng}$ TK-Renilla vector, and $4 \mu \mathrm{g}$ expression or control plasmids according to the manufacturer's instructions (Thermo Fisher Scientific, L3000015). At 36 hours after transfection, luciferase activity was measured with the Dual Luciferase Reporter Assay Kit (Promega, E2980). Expression of the reporter constructs was calculated as the fold induction over unstimulated transfected cells, using data from 3 independent experiments.

Transient overexpression in HEK293T, HaCaT cells, and primary keratinocytes. HEK293T cells (ACC 635, DSMZ Braunschweig, Germany) were transfected using $\mathrm{HeBS}$ buffer and $\mathrm{CaCl}_{2}$. $\mathrm{HaCaT}$ cells and primary keratinocytes were transfected with Lipofectamine 3000 according to the manufacturer's instructions (Thermo Fisher Scientific, L3000015). Five-microgram expression constructs were incubated with $3 \times 10^{5}$ cells for 4 hours. Thirty-six to 48 hours after transfection, cells were harvested and analyzed. NFKBIZ (catalog 44012), CDK9-HA (catalog 28102), and Flag-STAT3C (catalog 8722) expression constructs were purchased from Addgene and pINTOGFP-EZH2 T345A and pINTO-GFP-EZH2 T345D were provided by Danny Reinberg (Howard Hughes Medical Institute, New York University Langone Medical Center, New York, New York, USA).

Generation of STAT3 mutants. Mutation of STAT3 at K49, K140, and $\mathrm{K} 180$ was performed by site-directed mutagenesis of the human STAT3 pcDNA3 construct from Addgene (catalog 71447), which was previously cloned into the Strep-tagged backbone (pEXPR-IBA103). Substitution of the amino acid was performed with self-designed primers (Supplemental Table 1 and ref. 59).

CRISPR/Cas9 gene editing of STAT3 KO HaCaT cells. The CRISPR/ Cas9 one vector system was used to generated STAT3 KO HaCaT cells 
according to the protocol of Shalem et al. (60). The guide RNA against STAT3 (forward: 5'-CACCGACTGCTGGTCAATCTCTCCC-3', reverse: 5'-AAACGGGAGAGATTGACCAGCAGTC-3') was cloned into the Cas9 containing lentiCRISPRv2 containing Cas9 vector (Addgene, 52961), followed by lentiviral transduction and puromycin selection.

Synchronization of HaCaT cells. Synchronization of the cells with a double thymidine block was performed as described (61). After the second thymidine block, cells were released in normal medium. At $0,4,10$, and 14 hours after release, cells were stimulated with IL-36 $\alpha$ and/or abemaciclib for 1 hour. Propidium iodide staining was performed by flow cytometry (LSRII, Becton Dickinson) to detect the cell cycle phase at the time point of cell harvest.

Western blot analysis. Western blot analysis was performed as described (9). The following antibodies were used and purchased

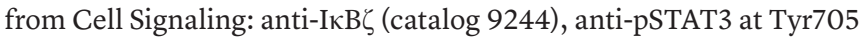
(catalog 9145), anti-pSTAT3 at Ser727 (catalog 9134), anti-STAT3 (catalog 12640), anti-p65 (catalog 8242), anti-EZH2 (catalog 5246), anti-pRB (at Ser807/811; catalog 8516), anti-FoxM1 (catalog 5436), anti-H3 (catalog 4499), anti-CDK4 (catalog 12790), anti-CDK6 (catalog 13331), anti-CDK9 (catalog 2316), anti-cyclin D1 (catalog 2978), anti-cyclin D2 (catalog 3741), anti-cyclin D3 (catalog 2936), anti-cJun (catalog 9165), anti-H3K27me3 (catalog 9733), anti-GAPDH (catalog 2118), anti-H3 (catalog 9715) and anti- $\beta$-actin (catalog 3700). Anti- $\alpha$-Tubulin (T9026) was purchased from MilliporeSigma. Anti- $\beta$-Gal (sc377257) and anti-GFP (sc9996) were obtained from Santa Cruz Biotechnology. Anti-pEZH2 at T345 (catalog 61242) and anti-pEZH2 at T487 (catalog 12820) were purchased from Active Motif and anti-pan-methyl-lysine antibody was purchased from Enzo (ADI-KAP-TF121-E). For detection of mouse I $\kappa \zeta$, a self-made rabbit antiserum raised against peptides CSAPGSPGSDSSDFSS and CLHIRSHKQKASGQ was applied (50).

Chromatin immunoprecipitation (ChIP). ChIP assays were performed as described (62). After sonification, chromatin was incubated with protein G-coupled Dynabeads (10004D, Invitrogen) and 2 $\mu \mathrm{g}$ STAT3 (Thermo Fisher Scientific, MA1-13042), CDK4 (Cell Signaling, 12790), CDK6 (MilliporeSigma, HPA002637), EZH2 (Diagenode, C15410039), NF-kB p65 (Diagenode, C15310256), or control IgG antibody (Abcam, ab46540) overnight at $4^{\circ} \mathrm{C}$. The promoter region of myoglobulin $(M B)$ served as an internal negative control (forward: 5'- CTCTGCTCCTTTGCCACAAC-3', reverse: 5'-GAGTGCTCTTCGGGTTTCAG-3'). ChIP primers corresponding to the promoter region of NFKBIZ (forward 5'-GCCTTAACTGGGCTAACAGC-3', reverse 5'-CTGGCAAGTCCTGGAAGGAG-3'), CCND2 (forward 5'-GGGAGAGGGAGGAGAGCTAA-3', reverse 5'-GAGAGGTGAGGGCAGAGAGA-3'), and CCND3 (forward 5'- GGCAATTACAGCCACATTCC-3', reverse 5'-GGTGGCAACAGACACTGCTA- $3^{\prime}$ ) were self-designed. Data from 2 independent experiments are presented as the fold enrichment, calculated over the percentage of input from the IgG control ChIP.

Coimmunoprecipitation (CoIP). Cells were lysed by mechanical disruption using a Dounce homogenizer and standard lysis buffer (50 mM Tris- $\mathrm{HCl} \mathrm{pH} 7.5,150 \mathrm{mM} \mathrm{NaCl}, 1 \% \mathrm{NP}-40,1 \mathrm{x}$ Protease inhibitor cocktail, Roche). Subsequently, lysates were sonicated for 5 minutes at high power (Bioruptor, Diagenode), followed by preclearing of the lysates with protein A/G PLUS agarose beads (Santa Cruz, sc-2003) for 1 hour at $4^{\circ} \mathrm{C}$. Precleared lysates were incubated either with antibodies specific for CDK4 (Cell Signaling, 12790),
CDK6 (MilliporeSigma, HPA002637), EZH2 (Cell Signaling, 5246), STAT3 (MA1-13042, Thermo Fisher Scientific) or $\beta$-Gal (sc-19119, Santa Cruz) as an IgG control, overnight at $4^{\circ} \mathrm{C}$. For endogenous IPs immune complexes were precipitated with protein A/G PLUS agarose beads and eluted by $6 \times$ SDS-PAGE sample buffer.

Cytokine array. Cytokine levels were detected from primary human keratinocytes that had been treated for 24 hours with 100 $\mathrm{ng} / \mathrm{mL}$ IL-36 $\alpha$, using the human cytokine array from R\&D Systems (ARYO05B) according to the manufacturer's instructions. Prior to analysis, input lysates were normalized to equal cell numbers. Spot intensity was quantified with the dot blot analyzer from ImageJ and normalized to the reference spots. Relative expression levels are represented as mean pixel intensities.

Gene expression analysis by $q P C R$. Gene expression analyses were performed as described (9). Relative gene expression was analyzed using self-designed primers ordered at Metabion (Supplemental Table 2). Relative mRNA levels were calculated by normalization to the human reference gene RPL37A or the mouse reference gene Actin using the $2-\Delta \Delta \mathrm{Ct}$ method.

Mice. Experiments were conducted in accordance with the German law guidelines of animal care. Ears of female C57BL/6 mice (8-12 weeks old, Jackson Laboratory) were topically treated for 6 consecutive days with $5 \mathrm{mg}$ Aldara cream (containing 5\% imiquimod, $3 \mathrm{M}$ Pharmaceuticals) and $10 \mu \mathrm{L}$ abemaciclib (2\% in $10 \mu \mathrm{L}$ ethanol), $10 \mu \mathrm{L}$ CPI-169 (5\% in $10 \mu \mathrm{L}$ ethanol), or vehicle control. At day 7 , mice were sacrificed and analyzed. In the therapeutic mouse model, IMQ was applied first to establish psoriasis-like skin lesions. Then, starting on the third day of IMQ administration (IMQ day 2), IMQ and the inhibitors were added in parallel until mice were sacrificed at day 6 (IMQ day 6). In the IL-36 $\alpha$-mediated psoriasis model, ears of male C57BL/6 mice (8-12 weeks old, Jackson Laboratory) were treated by intradermal injections of $1 \mu \mathrm{g}$ murine IL-36 $\alpha$ (7059-ML, R\&D Systems) or PBS control for 5 consecutive days. For application of abemaciclib ( $2 \%$ in ethanol), CPI-169 (5\% in ethanol), or the vehicle control, substances were mixed with Miglyol 812 (Carl Roth) in a ratio of 1:2. Inhibitors were topically applied 6 hours before intradermal injections of IL-36 $\alpha$ or PBS. Mice were sacrificed and analyzed at day 6. For treatment of established psoriasis-like skin disease, mice were treated every second day with IL-36 $\alpha$ for 8 days, followed by the analysis of the mice at day 9 (IL-36 day 9). Both abemaciclib and CPI-169 were applied daily on the skin, starting from the third IL-36 $\alpha$ administration (IL-36 day 4).

Flow cytometry. Sample preparation was performed as described (9). The following anti-mouse antibodies from BioLegend were used: anti-CD45 FITC (catalog 103107), anti-CD11b Pacific Blue (catalog 101223), anti-Ly6G PE (catalog 127607), anti-F4/80 APC (catalog 123115), anti-CD11c Pacific Blue (catalog 117322), antiMHC-II APC (catalog 107613), anti-CD172a PE (catalog 144011), and anti-Siglec-H PE (catalog 129605). Anti-PDCA-1 APC (172092-80) and anti- $\alpha \beta$ TCR Pacific Blue (catalog HM3628) were purchased from Invitrogen, and anti- $\gamma \delta$ TCR APC (catalog 17-5711-82) from MilliporeSigma. Acquisition was performed with the LSRII flow cytometer (Becton Dickinson) and live, single cells were gated using the FlowJo (Tree Star) software.

Histology. Ear sections from mice were fixed in $10 \%$ formalin (Carl Roth) and subsequently embedded in paraffin. Five-micrometer sections were prepared and incubated with the following antibodies from 
Cell Signaling: pSTAT3 (catalog 9145), pRB (catalog 8516), H3K27me3 (catalog 9733) and EZH2 (catalog 5246), cyclin D2 (catalog 3741), and cyclin D3 (catalog 2936). Antigen retrieval was performed in $1 \mathrm{mM}$ EDTA pH 8.0 for pSTAT3, and $10 \mathrm{mM}$ citrate buffer $\mathrm{pH} 6.0+0.5 \%$ Triton X-100 for EZH2, H3K27me3, pRB, cyclin D2, and cyclin D3. After incubation with peroxidase-coupled secondary antibodies, sections were stained with DAB substrate.

Analysis of patient data. Gene expression data originated from the GEO data set GSE13355 $(63,64)$. Prenormalized gene expression values from each sample were directly taken from the GEO profile data set GDS4602. The following reporters were taken for analysis: EZH2, ID 203358_s_at; CCND1, ID 208711_s_at; CCND2, ID 200953_s_at; and CCND3, ID 201700_s_at.

Statistics. Results from in vivo experiments are represented as the mean \pm SEM. Results from cell culture experiments are represented as the mean \pm SD. Significance was calculated using a 1-way ANOVA to compare multiple groups, and a 2-tailed Student's $t$ test was applied when 2 groups were compared with each other. A P value less than 0.05 was considered significant. Significance is depicted by asterisks as follows: ${ }^{*} P<0.05,{ }^{* *} P<0.01,{ }^{* * *} P<0.001$.

Study approval. All animal experiments were approved by the Regierungspräsidium, Tübingen, Germany (IB 4/18G, IB 1/19G). Human psoriasis skin samples came from the Department of Dermatology, Heidelberg University Hospital. Experiments were approved by the ethics committee of the University Hospital Heidelberg. Isolation of primary human keratinocytes from foreskin was approved by the local ethics committee of the University Hospital Tübingen.

\section{Author contributions}

$\mathrm{AM}, \mathrm{AD}$, and $\mathrm{CR}$ performed experiments and data analysis. $\mathrm{SH}$, $\mathrm{KSO}, \mathrm{MD}$, and DK designed the experiments. KS donated human psoriasis skin samples and helped with the analysis. $\mathrm{SH}, \mathrm{KSO}, \mathrm{MD}$, and DK wrote the manuscript.

\section{Acknowledgments}

We thank Michael Kracht for the CDK6 S178P construct and Danny Reinberg for the EZH2 T345A and T345D constructs. Several expression constructs were obtained from Addgene and provided by the following individuals: Sander van den Heuvel (CDK6, CDK4, CDK6DN), Andrew Rice (HA-CDK9), Kristian Helin (HA-EZH2), Jin Chen (cJUN), George Darnell (p65), Jim Darnell (STAT3C), Jie Chen (STAT3), Philip Hinds (cyclin D2), Bob Weinberg (cyclin D3), Bruce Zetter (cyclin D1), Feng Zhang (lentiCRISPRv2), and Stephen Elledge (pInducer20). We thank Caroline Schönfeld for technical assistance. The study was supported by grants from the Else-Kröner-Fresenius-Stiftung (to DK), the TR/SFB 156 (to DK and SH), the TR/SFB 209 (to $\mathrm{SH}$ and KSO), the DFG Excellent Strategy EXC-2180 and the Emmy-Noether program of the Deutsche Forschungsgemeinschaft (both to $\mathrm{SH}$ ).

Address correspondence to: Daniela Kramer, Interfaculty Institute for Biochemistry, Auf der Morgenstelle 34, 72076 Tübingen, Germany. Phone: 49.7071.2974159; Email: daniela.kramer@ uni-tuebingen.de.
1. Nestle FO, Kaplan DH, Barker J. Psoriasis. N Engl JMed. 2009;361(5):496-509.

2. Lowes MA, Suárez-Fariñas M, Krueger JG. Immunology of psoriasis. Annu Rev Immunol. 2014;32:227-255.

3. McGeachy MJ, Cua DJ, Gaffen SL. The IL-17 family of cytokines in health and disease. Immunity. 2019;50(4):892-906.

4. Kurschus FC, Moos S. IL-17 for therapy. J Dermatol Sci. 2017;87(3):221-227.

5. Wolf J, Ferris LK. Anti-IL-36R antibodies, potentially useful for the treatment of psoriasis: a patent evaluation of WO2013074569. Expert Opin Ther Pat. 2014;24(4):477-479.

6. Wasilewska A, Winiarska M, Olszewska M, Rudnicka L. Interleukin-17 inhibitors. A new era in treatment of psoriasis and other skin diseases. Postepy Dermatol Alergol. 2016;33(4):247-252.

7. Jullien D, Prinz JC, Nestle FO. Immunogenicity of biotherapy used in psoriasis: the science behind the scenes. J Invest Dermatol. 2015;135(1):31-38.

8. Johansen $\mathrm{C}$, et al. $\mathrm{I} \kappa \mathrm{B} \zeta$ is a key driver in the development of psoriasis. Proc Natl Acad Sci USA. 2015;112(43):E5825-E5833.

9. Müller $\mathrm{A}$, et al. IкB $\zeta$ is a key transcriptional regulator of IL-36-driven psoriasis-related gene expression in keratinocytes. Proc Natl Acad Sci USA. 2018;115(40):10088-10093.

10. Oeckinghaus A, Hayden MS, Ghosh S. Crosstalk in NF- $\kappa \mathrm{B}$ signaling pathways. Nat Immunol. 2011;12(8):695-708.

11. Zhang Q, et al. Tet2 is required to resolve inflammation by recruiting Hdac 2 to specifically repress IL-6. Nature. 2015;525(7569):389-393.
12. Tartey $S$, et al. Akirin 2 is critical for inducing inflammatory genes by bridging I $\mathrm{KB}-\zeta$ and the SWI/SNF complex. EMBO J. 2014;33(20):2332-2348.

13. Annemann $\mathrm{M}$, et al. Atypical I $\kappa \mathrm{B}$ proteins in immune cell differentiation and function. Immunol Lett. 2016;171:26-35.

14. Sherr CJ, Beach D, Shapiro GI. Targeting CDK4 and CDK6: from discovery to therapy. Cancer Discov. 2016;6(4):353-367.

15. Choi YJ, Anders L. Signaling through cyclin D-dependent kinases. Oncogene. 2014;33(15):1890-1903.

16. Gong X, et al. Genomic aberrations that activate D-type cyclins are associated with enhanced sensitivity to the CDK4 and CDK6 inhibitor abemaciclib. Cancer Cell. 2017;32(6):761-776.e6.

17. Vidula N, Rugo HS. Cyclin-dependent kinase $4 / 6$ inhibitors for the treatment of breast cancer: a review of preclinical and clinical data. Clin Breast Cancer. 2016;16(1):8-17.

18. Klein ME, Kovatcheva M, Davis LE, Tap WD, Koff A. CDK4/6 inhibitors: the mechanism of action may not be as simple as once thought. Cancer Cell. 2018;34(1):9-20.

19. Goel S, et al. CDK4/6 inhibition triggers anti-tumour immunity. Nature. 2017;548(7668):471-475

20. Amulic B, et al. Cell-cycle proteins control production of neutrophil extracellular traps. Dev Cell. 2017;43(4):449-462.e5.

21. Scheicher R, et al. CDK6 as a key regulator of hematopoietic and leukemic stem cell activation. Blood. 2015;125(1):90-101.

22. Schaer DA, et al. The CDK4/6 inhibitor abemaciclib induces a $\mathrm{T}$ cell inflamed tumor microenviron- ment and enhances the efficacy of PD-L1 checkpoint blockade. Cell Rep. 2018;22(11):2978-2994.

23. Kollmann $\mathrm{K}$, et al. A kinase-independent function of CDK6 links the cell cycle to tumor angiogenesis. Cancer Cell. 2013;24(2):167-181.

24. Buss H, et al. Cyclin-dependent kinase 6 phosphorylates NF-кB P65 at serine 536 and contributes to the regulation of inflammatory gene expression. PLOS ONE. 2012;7(12):e51847.

25. Handschick K, et al. Cyclin-dependent kinase 6 is a chromatin-bound cofactor for NF- $\mathrm{KB}$-dependent gene expression. Mol Cell. 2014;53(2):193-208.

26. Moos S, Mohebiany AN, Waisman A, Kurschus FC. Imiquimod-induced psoriasis in mice depends on the IL-17 signaling of keratinocytes. J Invest Dermatol. 2019;139(5):1110-1117.

27. Tortola L, et al. Psoriasiform dermatitis is driven by IL-36-mediated DC-keratinocyte crosstalk. J Clin Invest. 2012;122(11):3965-3976.

28. Iwanaga R, et al. Activation of the cyclin D2 and cdk6 genes through NF-kappaB is critical for cell-cycle progression induced by HTLV-I Tax. Oncogene. 2008;27(42):5635-5642.

29. Wang Z, Sicinski P, Weinberg RA, Zhang Y, Ravid K. Characterization of the mouse cyclin D3 gene: exon/intron organization and promoter activity. Genomics. 1996;35(1):156-163.

30. Shi X, Zhang H, Paddon H, Lee G, Cao X, Pelech S. Phosphorylation of STAT3 serine-727 by cyclin-dependent kinase 1 is critical for nocodazole-induced mitotic arrest. Biochemistry. 2006;45(18):5857-5867.

31. Ezhkova E, et al. Ezh2 orchestrates gene expression for the stepwise differentiation of 
tissue-specific stem cells. Cell. 2009;136(6):1122-1135.

32. Eckert RL, Adhikary G, Rorke EA, Chew YC, Balasubramanian S. Polycomb group proteins are key regulators of keratinocyte function. J Invest Dermatol. 2011;131(2):295-301.

33. Wurm $\mathrm{S}$, et al. Terminal epidermal differentiation is regulated by the interaction of Fra-2/AP-1 with Ezh2 and ERK1/2. Genes Dev. 2015;29(2):144-156.

34. Kim E, et al. Phosphorylation of EZH2 activates STAT3 signaling via STAT3 methylation and promotes tumorigenicity of glioblastoma stem-like cells. Cancer Cell. 2013;23(6):839-852.

35. Dasgupta M, Dermawan JK, Willard B, Stark GR. STAT3-driven transcription depends upon the dimethylation of K49 by EZH2. Proc Natl Acad Sci USA. 2015;112(13):3985-3990.

36. Yang J, et al. Reversible methylation of promoter-bound STAT3 by histone-modifying enzymes. Proc Natl Acad Sci USA. 2010;107(50):21499-21504.

37. Iannetti $A$, et al. Regulation of $\mathrm{p} 53$ and $\mathrm{Rb}$ links the alternative NF- $\mathrm{kB}$ pathway to EZH2 expression and cell senescence. PLoS Genet. 2014;10(9):e1004642.

38. Zeng X, Chen S, Huang H. Phosphorylation of EZH2 by CDK1 and CDK2: a possible regulatory mechanism of transmission of the H3K27me3 epigenetic mark through cell divisions. Cell Cycle. 2011;10(4):579-583.

39. Chen S, et al. Cyclin-dependent kinases regulate epigenetic gene silencing through phosphorylation of EZH2. Nat Cell Biol. 2010;12(11):1108-1114.

40. Wei Y, et al. CDK1-dependent phosphorylation of EZH2 suppresses methylation of H3K27 and promotes osteogenic differentiation of human mesenchymal stem cells. Nat Cell Biol. 2011;13(1):87-94.

41. Wolk K, et al. IL-22 and IL-20 are key mediators of the epidermal alterations in psoriasis while IL-17 and IFN-gamma are not. J Mol Med. 2009;87(5):523-536.
42. Zheng Y, et al. Interleukin-22, a T(H)17 cytokine, mediates IL-23-induced dermal inflammation and acanthosis. Nature. 2007;445(7128):648-651.

43. Calautti E, Avalle L, Poli V. Psoriasis: A STAT3-centric view. Int J Mol Sci. 2018;19(1):E171.

44. Bradley WD, et al. EZH2 inhibitor efficacy in non-Hodgkin's lymphoma does not require suppression of H3K27 monomethylation. Chem Biol. 2014;21(11):1463-1475.

45. van der Fits L, et al. Imiquimod-induced psoriasis-like skin inflammation in mice is mediated via the IL-23/IL-17 axis. J Immunol. 2009;182(9):5836-5845.

46. Anders L, et al. A systematic screen for CDK4/6 substrates links FOXM1 phosphorylation to senescence suppression in cancer cells. Cancer Cell. 2011;20(5):620-634.

47. Campbell JJ, et al. Efficacy of chemokine receptor inhibition in treating IL-36 $\alpha$-induced psoriasiform inflammation. JImmunol. 2019;202(6):1687-1692.

48. Laphanuwat $P$, Jirawatnotai S. Immunomodulatory roles of cell cycle regulators. Front Cell Dev Biol. 2019;7:23.

49. Tsoi LC, et al. Enhanced meta-analysis and replication studies identify five new psoriasis susceptibility loci. Nat Commun. 2015;6:7001.

50. Lorscheid S, et al. Keratinocyte-derived IкB $\zeta$ drives psoriasis and associated systemic inflammation. JCI Insight. 2019;4(22):130835.

51. Li J, et al. TRIM28 interacts with EZH2 and SWI/ SNF to activate genes that promote mammosphere formation. Oncogene. 2017;36(21):2991-3001.

52. Shi B, et al. Integration of estrogen and Wnt signaling circuits by the polycomb group protein EZH2 in breast cancer cells. Mol Cell Biol. 2007;27(14):5105-5119.

53. Kaneko S, et al. Phosphorylation of the PRC2 component Ezh2 is cell cycle-regulated and up-regulates its binding to ncRNA. Genes Dev. 2010;24(23):2615-2620.
54. Kim J, et al. Polycomb- and methylation-independent roles of EZH2 as a Transcription Activator. Cell Rep. 2018;25(10):2808-2820.e4.

55. Lee ST, et al. Context-specific regulation of NF-kB target gene expression by EZH2 in breast cancers. Mol Cell. 2011;43(5):798-810.

56. Miyoshi K, et al. Stat 3 as a therapeutic target for the treatment of psoriasis: a clinical feasibility study with STA-21, a Stat3 inhibitor. J Invest Dermatol. 2011;131(1):108-117.

57. Boukamp P, Petrussevska RT, Breitkreutz D, Hornung J, Markham A, Fusenig NE. Normal keratinization in a spontaneously immortalized aneuploid human keratinocyte cell line. JCell Biol.1988;106(3):761-771.

58. Sambrook J, Russell DW. Calcium-phosphate-mediated transfection of eukaryotic cells with plasmid DNAs. CSH Protoc. 2006;2006(1):pdb.prot3871.

59. Zheng L, Baumann U, Reymond JL. An efficient one-step site-directed and site-saturation mutagenesis protocol. Nucleic Acids Res. 2004;32(14):e115.

60. Shalem O, et al. Genome-scale CRISPR-Cas9 knockout screening in human cells. Science. 2014;343(6166):84-87.

61. Peña-Diaz J, et al. Transcription profiling during the cell cycle shows that a subset of Polycomb-targeted genes is upregulated during DNA replication. Nucleic Acids Res. 2013;41(5):2846-2856.

62. Nemajerova A, et al. TAp73 is a central transcriptional regulator of airway multiciliogenesis. Genes Dev. 2016;30(11):1300-1312.

63. Nair RP, et al. Genome-wide scan reveals association of psoriasis with IL-23 and NF-kappaB pathways. Nat Genet. 2009;41(2):199-204.

64. Swindell WR, et al. Genome-wide expression profiling of five mouse models identifies similarities and differences with human psoriasis. PLOS ONE. 2011;6(4):e18266. 